Article

\title{
Synthesis and Chemiluminescent Properties of Amino-Acylated luminol Derivatives Bearing Phosphonium Cations
}

\author{
Anna Pantelia ${ }^{1}$, Ira Daskalaki ${ }^{1}$, M. Consuelo Cuquerella ${ }^{2}$, Georgios Rotas ${ }^{1}$, \\ Miguel A. Miranda ${ }^{2, *}$ and Georgios C. Vougioukalakis ${ }^{1, * \mathbb{D}}$ \\ 1 Laboratory of Organic Chemistry, Department of Chemistry, National and Kapodistrian University of \\ Athens, Panepistimiopolis, 15771 Athens, Greece; annapantelia@gmail.com (A.P.); \\ iradask@hotmail.com (I.D.); rotasgiorgos@hotmail.com (G.R.) \\ 2 Instituto de Tecnología Química (UPV-CSIC), Universitat Politècnica de València, 46022 València, Spain; \\ mcuquere@itq.upv.es \\ * Correspondence: mmiranda@qim.upv.es (M.A.M.); vougiouk@chem.uoa.gr (G.C.V.); \\ Tel.: +34-963-877-807 (M.A.M.); +30-210-727-4230 (G.C.V.); \\ Fax: +34-963-879-444 (M.A.M.); +30-210-727-4761 (G.C.V.)
}

Received: 15 October 2019; Accepted: 30 October 2019; Published: 31 October 2019

\begin{abstract}
The monitoring of reactive oxygen species in living cells provides valuable information on cell function and performance. Lately, the development of chemiluminescence-based reactive oxygen species monitoring has gained increased attention due to the advantages posed by chemiluminescence, including its rapid measurement and high sensitivity. In this respect, specific organelle-targeting trackers with strong chemiluminescence performance are of high importance. We herein report the synthesis and chemiluminescence properties of eight novel phosphonium-functionalized amino-acylated luminol and isoluminol derivatives, designed as mitochondriotropic chemiluminescence reactive oxygen species trackers. Three different phosphonium cationic moieties were employed (phenyl, p-tolyl, and cyclohexyl), as well as two alkanoyl chains (hexanoyl and undecanoyl) as bridges/linkers. Synthesis is accomplished via the acylation of the corresponding phthalimides, as phthalhydrazide precursors, followed by hydrazinolysis. This method was chosen because the direct acylation of (iso)luminol was discouraging. The new derivatives' chemiluminescence was evaluated and compared with that of the parent molecules. A relatively poor chemiluminescence performance was observed for all derivatives, with the isoluminol-based ones being the poorest. This result is mainly attributed to the low yield of the fluorescence species formation during the chemiluminescence oxidation reaction.
\end{abstract}

Keywords: phthalhydrazide; luminol; chemiluminescence; peroxide; phosphonium; mitochondria

\section{Introduction}

Chemiluminescence (CL), the emission of light derived from a chemical reaction, is usually the outcome of a substrate's redox reaction towards the formation of an excited species, which emits light upon deactivation [1]. This chemically-induced light generation is of high importance, for both detection and analytical purposes, finding applications in analytical chemistry, clinical diagnostics, forensics, etc. [2-4]. High sensitivity, linear response, and fast measurement are among the main advantages of CL-based analyses. In parallel, the growing evidence on the importance of certain highly-reactive oxidants, known as reactive oxygen and nitrogen species (ROS and RNS, respectively), in cell signaling, homeostasis, and metabolism [5-8] has necessitated the development of methods for detecting the intracellular levels of ROS/RNS $[9,10]$. In this regard, mitochondria have been identified 
as the primary ROS-producing organelles [11-13]. The existence of ROS/RNS, both ideal oxidants in CL reactions, has led to the use of chemiluminescent ROS-detecting probes, capitalizing on the advantages of CL-based analyses [14-18].

5-Amino-2,3-dihydrophthalazine-1,4-dione is probably the most notorious and widely-used chemiluminescent reagent [19-21]. It was synthesized for the first time at the beginning of the 20th century [22], but its outstanding CL properties were discovered 26 years later [23]. The nickname "Luminol" was given to this bicyclic compound, due to its intriguing CL properties [24], while fame came a little later, when it was first reported as an efficient blood tracker in forensics [25]. Luminol's strong chemiluminescence is triggered upon oxidation from peroxide in the presence of a catalyst (peroxidases, $\mathrm{Fe}^{3+}, \mathrm{HOCl}$ ), yielding the excited 3-aminophthalate anion, which has been identified as the light-emitting species. Although its laboratory use is widespread, it has been only recently employed as in vivo CL tracker of neutrophil anti-microbial activity, either unmodified [26,27], or as a functional biodegradable material [28]. In this respect, functionalization of luminol with targeting moieties is expected to result in novel organelle-specific molecular trackers.

Our present work is part of an ongoing collaborative project on the development of novel, mitochondriotropic chemiluminescent probes for ROS detection. In this regard, we opt for the synthesis of tailor-designed luminol and isoluminol (the 6-amino isomer of luminol) derivatives, covalently linked with phosphonium cations as mitochondriotropic moieties [29-31]. Herein, we report on the synthesis and chemiluminescent properties of amino-acylated luminol and isoluminol derivatives bearing variable phosphonium cations (triphenyl, tris(4-tolyl) or tricyclohexyl) and chain lengths (hexyl, undecyl). Amino-acylation of (iso)luminol was chosen due to the seemingly ease of synthesis. Despite luminol's high repute, chemically modified derivatives are rather limited. Simple acylated luminol derivatives have been synthesized and their chemiluminescence efficiencies have been evaluated more than 50 years ago [32-34]. Their synthesis was reportedly performed via the direct acylation of luminol with acyl chlorides, while their CL efficiencies appeared to be much lower to those of luminol, indicating that chemical functionalization can substantially alter the CL properties of the parent molecule. On the other hand, highly-efficient (electro)chemiluminescent luminol-Ru(bpy) 3 donor-acceptor dyads $[35,36]$ have been lately prepared from the direct acylation of luminol, while acylation has very recently been also achieved using cyclic anhydrides [37]. Thus, our goal was two-fold, namely: a) the development of an efficient synthetic procedure for a series of luminol and isoluminol amino-acylated phosphonium derivatives; and b) the evaluation of the chemiluminescence properties of these derivatives, in order to assess their potential for in vivo CL performance. Along these lines, a re-evaluation of the CL properties of acyl-(iso)luminol derivatives, studied by modern spectroscopic techniques, is presented herein.

\section{Results}

\subsection{Synthesis of the Target Compounds}

The synthesis of amino-acylated luminol derivatives $\mathbf{1}$ was initially approached through the direct acylation of luminol (Lum) with a phosphonium-carboxylic acid derivative, albeit without success (Scheme 1). A variety of methods were employed, involving the reaction of luminol with phosphonium alkanoic acids $\mathbf{2 a}$ or $\mathbf{2} \mathbf{b}$, (prepared from the reaction of 6-bromo-hexanoic or undecanoic acid with triphenylphosphine) [38] through acid chloride [32], or using coupling agents, yielding, in both cases, inseparable mixtures from which the desired product 1 could not be isolated in sufficient purity and yield. Other approaches, involving the use of NHS-activated esters, anhydride (prepared from DCC-mediated condensation of 2 [39]), or mixed anhydride (prepared in situ from 2 using ethyl chloroformate [40]), yielded again either inseparable mixtures, or no reaction. Preparation of $\mathbf{1}$ was also attempted using bromoalkanoic acids, followed by their reaction with phosphine, again without success. 


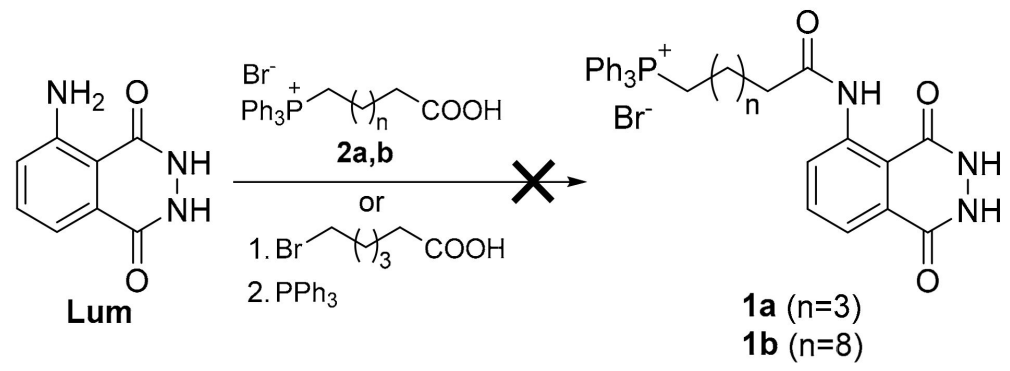

Scheme 1. Attempted direct acylation of luminol.

The above disappointing results led to another synthetic approach. Both the acidity of luminol's hydrazide protons and the weak nucleophilicity of its amino group were identified as potential source of byproducts (through 2-N-, 3-N-, or O-acylation). Therefore, amino-acylation was attempted on protected luminol derivatives. Phthalimides have been used as protected phthalhydrazides in the preparation of amino-alkylated isoluminol derivatives [41-43]. In this respect, amino-phthalimides $\mathbf{6} \mathbf{a}, \mathbf{b}$ (Scheme 2) were prepared from the respective nitrophthalic acids $\mathbf{3 a}, \mathbf{b}$ in a three-step reaction sequence involving consecutive condensation reactions towards anhydrides 4 and then phthalimides $\mathbf{5}$, and finally reduction of the nitro group [41,44]. Phthalimides $\mathbf{6 a}, \mathbf{b}$ show good solubility in common organic solvents and thus can be handled easier, as compared to the respective phthalhydrazides. Additionally, their easy and scalable preparation (no column chromatography needed) render them valuable intermediates in the synthesis of phthalhydrazide derivatives.

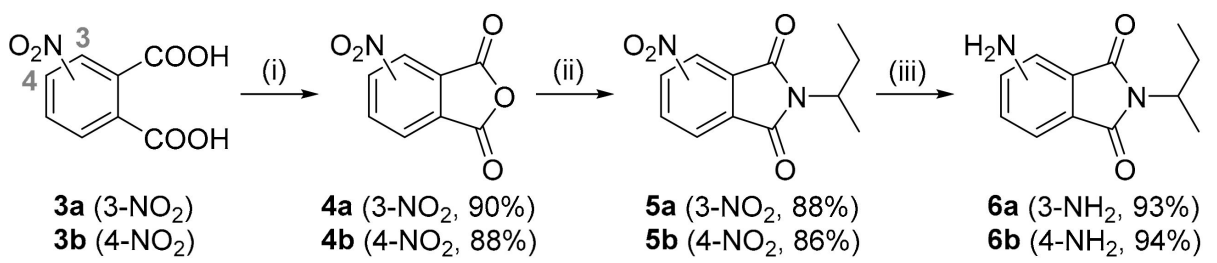

Scheme 2. Synthesis of aminophthalimides 6a,b. Reagents and conditions: (i) $\mathrm{Ac}_{2} \mathrm{O}, \Delta$; (ii) $s e c-\mathrm{BuNH}_{2}$, $\mathrm{AcOH}, \Delta$; (iii) $\mathrm{H}_{2}, \mathrm{Pd} / \mathrm{C}, \mathrm{MeOH}$.

Acylation of phthalimides $\mathbf{6 a}, \mathbf{b}$ proceeds smoothly with bromoalkyl carboxylic acids 7 via acyl chloride, furnishing the acylated phthalimides in moderate yields (Scheme 3). It is worth noting that room temperature has to be maintained throughout the reaction (even during the evaporation of oxalyl chloride), since halogen exchange occurs to some extent, towards the chloride, while more complex byproducts (e.g., 13 [45]) are isolated on prolonged heating. On the other hand, no reaction occurred when the acylation was attempted with the aid of coupling reagents ( $\mathrm{N}$-(3-dimethylaminopropyl)- $\mathrm{N}^{\prime}$-ethylcarbodiimide hydrochloride (EDC), $\mathrm{N}, \mathrm{N}^{\prime}$-Dicyclohexylcarbodiimide (DCC) or vanillin, 4-dimethylaminopyridine (DMAP)). This result was in stark contrast to that of the similar coupling reaction of luminol mentioned above, where complex mixtures were formed. This is a clear indication that the hydrazide group is the source of by-product(s) formation. Next, introduction of the appropriate phosphine was performed in refluxing acetonitrile, yielding the corresponding phosphonium cations in moderate to good yields. Tricyclohexylphosphonium derivative $\mathbf{1 1} \mathbf{b}$ was isolated as mixture with tricyclohexylphosphinoxide and was used in the next step as such. Phosphonium 1a has been also prepared from the direct acylation of phthalimide 6a with phosphonium carboxylic acid 2a (via acyl chloride) in $61 \%$ yield, rendering this procedure a good alternative. Finally, hydrazinolysis of the phthalimides afforded the desired phthalhydrazides $\mathbf{1}$ and $\mathbf{1 2}$ in moderate (non-optimized) yields. Prolonged reaction times and high temperatures during hydrazinolysis have to be avoided in order to bypass amide bond cleavage. 


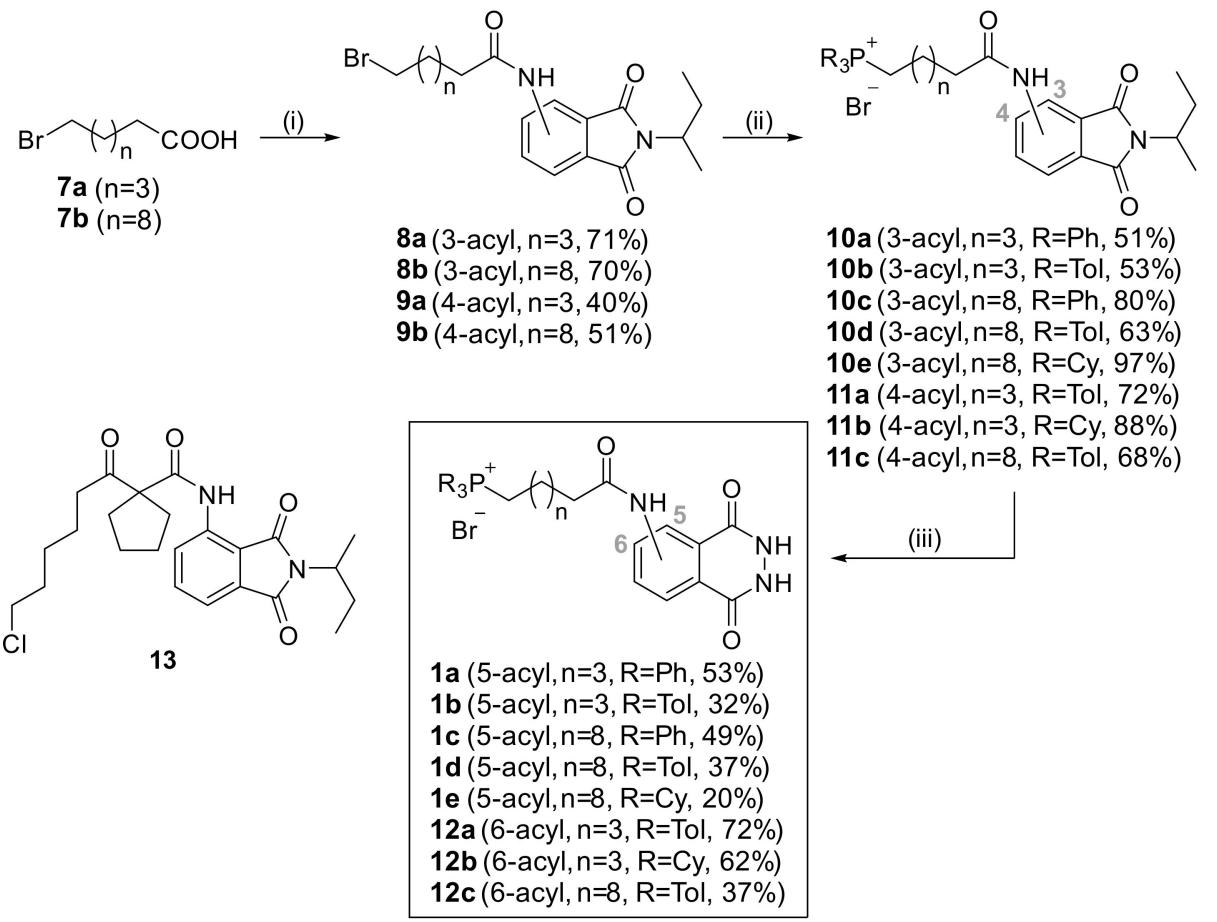

Scheme 3. Synthesis of phosphonium bearing phthalhydrazides. Reagents and conditions: (i) (a) $(\mathrm{COCl})_{2}$, (b) $6 \mathbf{a}$ or $6 \mathrm{~b}, \mathrm{Py}, \mathrm{DCM}$; (ii) $\mathrm{PR}_{3}, \mathrm{MeCN}, \Delta$; (iii) $\mathrm{NH}_{2} \mathrm{NH}_{2} \cdot \mathrm{H}_{2} \mathrm{O}, \mathrm{EtOH}, \Delta$. Yields are non-optimized.

The desired products can be thus isolated in a repeatable manner and employing the usual purification procedures. Acylation of luminol and isoluminol is evident in the ${ }^{1} \mathrm{H}-\mathrm{NMR}$ spectra of the derivatives, where characteristic patterns appear, as shown in Figure 1. All signals are shifted downfield, as compared to their parent compounds, while the newly-obtained amide NH protons appear quite deshielded. The hydrazide proton signals are not always evident, usually appearing as very broad peaks of variable chemical shift (12-8 ppm), quite sensitive to moisture, solvent traces, and sample concentrations.

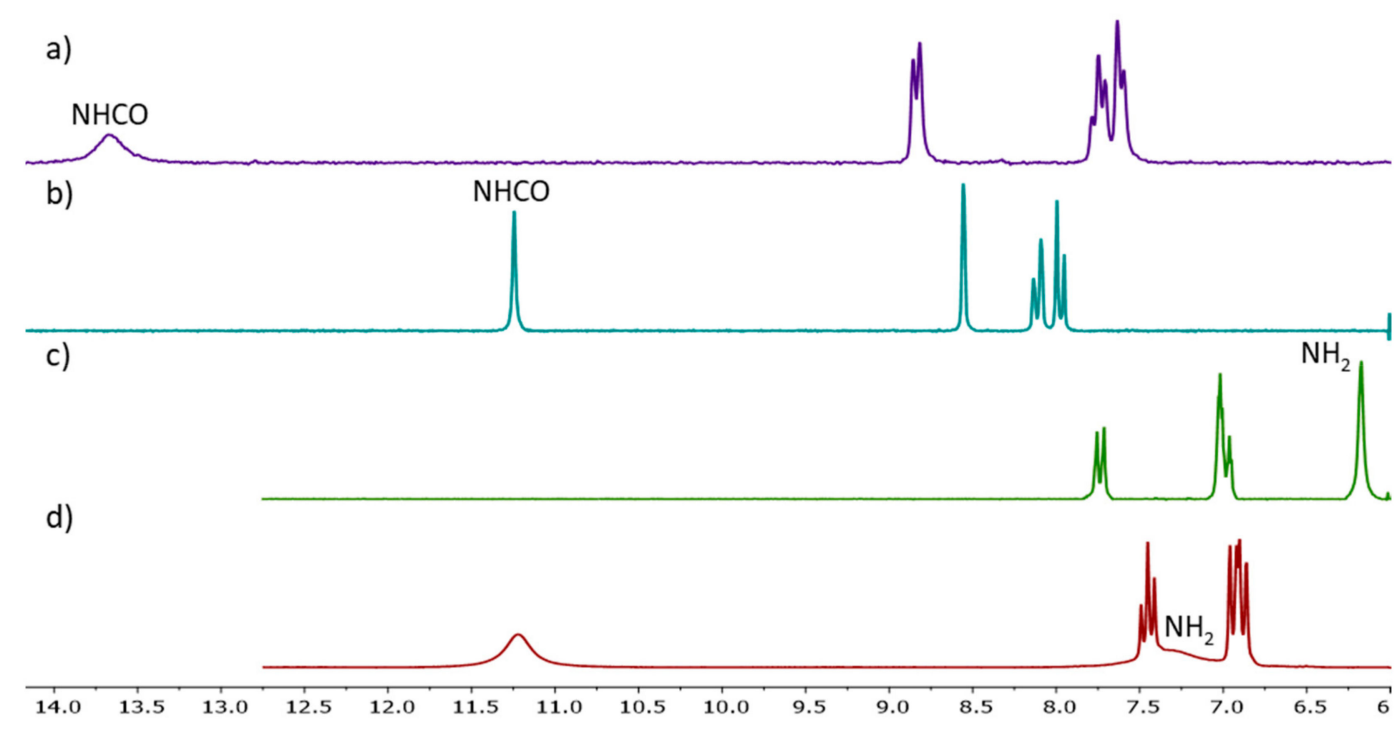

Figure 1. ${ }^{1} \mathrm{H}-\mathrm{NMR}\left(200 \mathrm{MHz}, \mathrm{DMSO}-d_{6}\right)$ spectra of: (a) 1e; (b) 12b; (c) isoluminol; and (d) luminol (aromatic region). The signals attributed to $5-\mathrm{N}$-amide $(\mathbf{a}, \mathbf{b})$ and amine $(\mathbf{c}, \mathbf{d})$ protons are indicated. 


\subsection{Chemiluminescence Studies}

In order to investigate the CL properties of the new luminol-phosphonium derivatives, a protocol was established. Briefly, (iso)luminols were dissolved in aqueous basic solutions, giving a final concentration of $7.5 \mu \mathrm{M}$. Then, each sample was introduced in a quartz cuvette and the CL was triggered by subsequent addition of $\mathrm{H}_{2} \mathrm{O}_{2}$ and $\mathrm{K}_{3}\left[\mathrm{Fe}(\mathrm{CN})_{6}\right]$ while vigorously stirring. Monitoring of the process was performed using a fluorometer with its own lamp switched off, running in the time-based mode. The CL displayed by the amino-acylated derivatives $\mathbf{1}$ and 12, under these experimental conditions, together with that of the parent compound luminol, are shown in Figure 2 and Table 1.

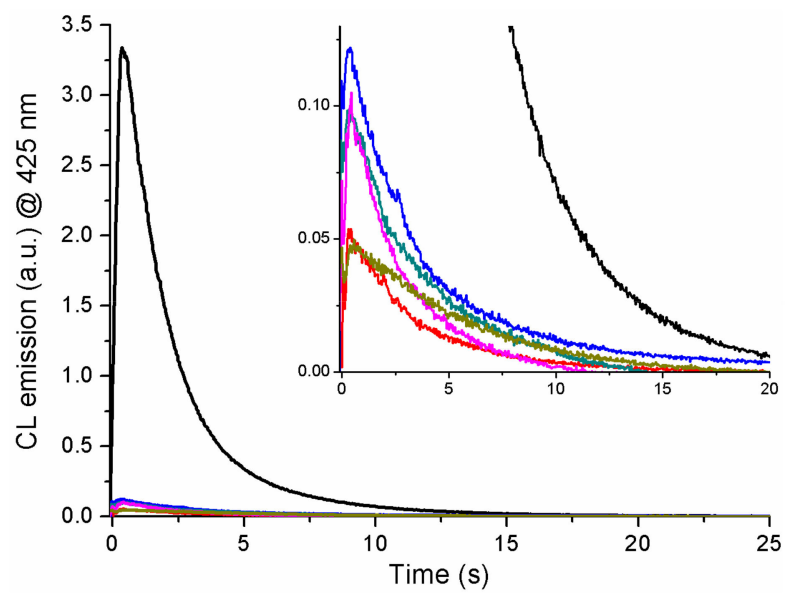

Figure 2. Results of a typical experiment to determine the chemiluminescence of the amino-acylated derivatives 1a (blue), $\mathbf{1 b}$ (green), 1c (red), 1d (magenta), 1e (olive), and luminol (black) in aqueous basic solutions. Inset: magnification.

Table 1. CL quantum yields of phthalhydrazides 1, 12, and luminol

\begin{tabular}{cc}
\hline Compound & $\boldsymbol{\Phi}_{\mathrm{CL}}$ \\
\hline Luminol & $0.012^{\mathrm{a}}$ \\
$\mathbf{1 a}$ & 0.001 \\
$\mathbf{1 b}$ & 0.001 \\
$\mathbf{1 c}$ & 0.001 \\
$\mathbf{1 d}$ & 0.001 \\
$\mathbf{1 e}$ & 0.001 \\
$\mathbf{1 2 a}$ & $<0.001$ \\
$\mathbf{1 2 b}$ & $<0.001$ \\
$\mathbf{1 2 c}$ & $<0.001$ \\
\hline \multicolumn{2}{c}{}
\end{tabular}

As a general finding, acylation diminishes almost quantitatively luminol's CL, irrespective of the mitotropic moieties incorporated to the structure, or the length of the chain employed to link them to the amino acylated luminols. Amino-acylated isoluminols (compounds 12) showed even a more dramatic effect, as CL was practically undetectable. Chemiluminescence quantum yields $\left(\Phi_{\mathrm{CL}}\right)$ were determined by comparison with luminol, taking its previously established absolute $\Phi^{0} \mathrm{CL}$ as 0.012 [46]. To do so, the total area under the curve for each compound was measured 10 times, in separate experiments, and the average value was used to calculate the relative $\Phi_{\mathrm{CL}}$ as $\Phi_{\mathrm{CL}}=\mathrm{A} / \mathrm{A}_{\mathrm{L}} \mathrm{x}$ $\Phi^{0} \mathrm{CL}$, where A corresponds to the average area for each compound and $\mathrm{A}_{\mathrm{L}}$ the value obtained in the case of the reference compound luminol. The results are listed in Table 1.

It is widely accepted that the oxidation of luminol leads to the formation of 3-aminophthalate (3AP) in its excited singlet state (Scheme 4 , step A). In part, this excited species $\left({ }^{1} \mathbf{3 A P} \mathbf{P}^{*}\right)$ relaxes to its 
ground state through the emission of light at $425 \mathrm{~nm}$ (Scheme 4, step B), thus producing the observed chemiluminescence in the global process. Taking this into account, the dramatic decrease of CL upon acylation could originate by a diminution in the yield of the oxidation and/or the emission steps. To ascertain which is the key step affected, the fluorescence of 3-heptanamidophthalic acid $\mathbf{1 4}$ was measured as reference compound (closely related to the emitting species in the CL of $\mathbf{1}$, prepared in two steps from 3-nitrophthalic acid) and compared to that of 3APH (Figure 3a) [47]. To this end, two alkaline isoabsorptive solutions of $\mathbf{3 A P H}$ and $\mathbf{1 4}$ were excited at the same wavelength $\left(\lambda_{\mathrm{exc}}=303 \mathrm{~nm}\right)$ to ensure that both absorb the same number of photons. The fluorescence spectra were then recorded and revealed that the emission quantum yields are of the same order, albeit significantly different $\left(\Phi_{\mathrm{F}}=0.17\right.$ for 14, as compared with $\Phi_{\mathrm{F}}=0.30$ [48] for the reference compound 3APH). This sole parameter does not justify the remarkable CL variations, which have to be attributed to the lower yield of aminophthalate formation from the oxidation reaction, possibly due to the lower electron donating capability of the aromatic ring substituent. Accordingly, the perturbation of the electronic distribution of the benzenoic chromophore is reflected in the significant changes observed in the UV-vis spectra (Figure 3b).

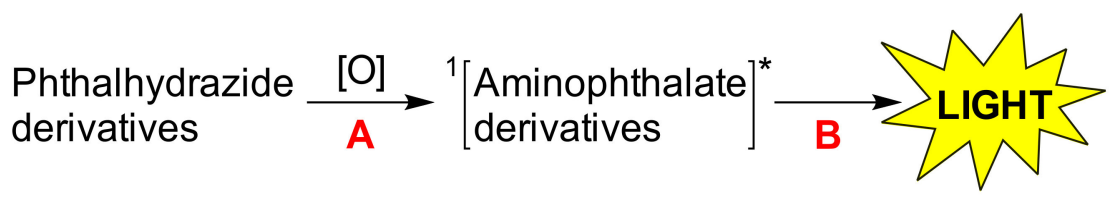

Scheme 4. Steps involved in phthalhydrazide derivatives' chemiluminescence.
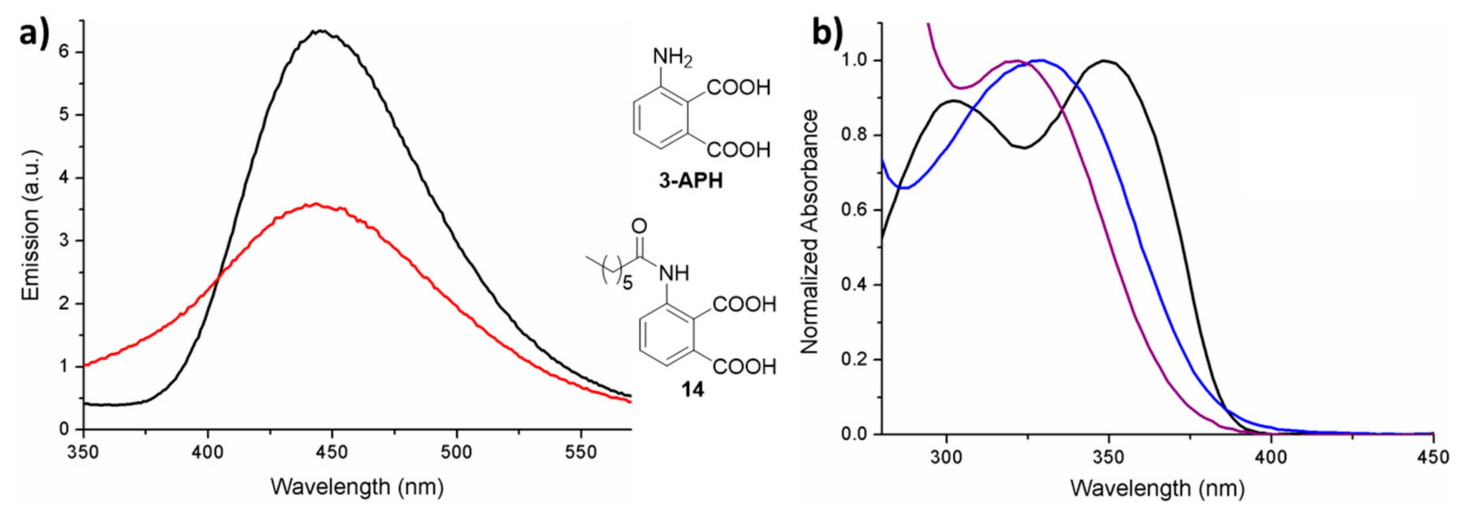

Figure 3. (a) Fluorescence spectra of 3 APH (black) and 14 (red) $\left(\lambda_{\text {exc }}=303 \mathrm{~nm}\right.$, matching absorbance) in aqueous basic solutions. (b) Normalized UV-vis spectra of luminol (black), 1a (blue) and 12a (violet) in aqueous basic solutions.

\section{Materials and Methods}

\subsection{General Information}

All chemicals were obtained from commercial sources and were used without further purification. Solvents were dried according to published procedures [49]. The course of the reactions was followed with thin-layer chromatography (TLC), using aluminum sheets $(0.2 \mathrm{~mm})$ coated with silica gel 60 with fluorescence indicator (silica gel 60 F254). Purification of the products was carried out by flash column chromatography, using silica gel 60 (230-400 mesh). Nuclear magnetic resonance (NMR) spectra were obtained with a Bruker Avance 400MHz (Bruker BioSpin MRI GmbH, Ettlingen, Germany) or a Varian Mercury 200MHz spectrometer (Varian Inc., Yarnton, UK). Chemical shifts are reported in ppm. HRMS spectra were recorded in a QTOF maXis impact (Bruker) spectrometer under electron spray ionization (ESI) conditions. Fluorescence spectra were registered with a Photon Technology International (PTI) spectrofluorometer (Photon Technology International, Inc. NJ, USA), model LPS-220B equipped with a $75 \mathrm{~W}$ Xe lamp as a light source, also equipped with a monochromator. Monitoring of the CL was 
performed using the same spectrofluorometer with its own lamp switched off. The set was run in the timebased mode with the detection dialed at $425 \mathrm{~nm}$. Each experiment was performed at least 10 times. Triggering the chemiluminescence: luminols were dissolved in aqueous basic solutions giving a final concentration of $7.5 \mu \mathrm{M}$. Then, $2 \mathrm{~mL}$ of each sample were introduced in a quartz cuvette and the CL was triggered by addition of $2.5 \mu \mathrm{L}$ of $\mathrm{H}_{2} \mathrm{O}_{2}(50 \% w / w)$ and $8 \mu \mathrm{L}$ of $\mathrm{K}_{3}\left[\mathrm{Fe}(\mathrm{CN})_{6}\right] 75 \mathrm{mM}$ while vigorously stirring. The ${ }^{1} \mathrm{H},{ }^{13} \mathrm{C}$ and ${ }^{31} \mathrm{P}$ NMR spectra of some compounds are in the Supplementary Materials.

\subsection{Synthetic Procedures}

\subsubsection{Synthesis of TPP Carboxylic Acids}

Triphenylphosphine (11.5 mmol) was added in a solution of bromoalkyl carboxylic acid (12 mmol) in dry acetonitrile $(20 \mathrm{~mL})$ and the mixture was stirred at reflux under argon for $48 \mathrm{~h}$. After cooling, the solvent was evaporated and the product precipitated out upon addition of ethyl acetate (or diethyl ether). Filtration and washing with the same solvent furnished pure products [38].

(5-Carboxypentyl)triphenylphosphonium bromide (2a). White powder (4.83 g, 92\%). ${ }^{1} \mathrm{H}$ NMR $\left(200 \mathrm{MHz}, \mathrm{CDCl}_{3}\right) \delta: 8.46(\mathrm{bs}, 1 \mathrm{H}, \mathrm{COOH}), 7.91-7.57(\mathrm{~m}, 15 \mathrm{H}, \mathrm{ArH}), 3.73-3.56\left(\mathrm{~m}, 2 \mathrm{H}, \mathrm{CH}_{2} \mathrm{P}\right), 2.41-2.33$ $\left(\mathrm{m}, 2 \mathrm{H}, \mathrm{CH}_{2} \mathrm{COOH}\right), 1.67-1.55\left(\mathrm{~m}, 6 \mathrm{H}, \mathrm{CH}_{2}\right) .{ }^{13} \mathrm{C} \mathrm{NMR}\left(50 \mathrm{MHz}, \mathrm{CDCl}_{3}\right) \delta: 176.09,135.21(\mathrm{~d}, J=2.8 \mathrm{~Hz}$, $\mathrm{PPh}_{3}$ para), 133.66 (d, J = 10.0 Hz, $\mathrm{PPh}_{3}$ ortho), 130.65 (d, $J=12.5 \mathrm{~Hz}, \mathrm{PPh}_{3}$ meta), 118.11 (d, $J=86.0 \mathrm{~Hz}$, $\mathrm{PPh}_{3}$ ipso), 34.25, 29.58 (d, $\left.J=16.2 \mathrm{~Hz}, \mathrm{CH}_{2} \mathrm{CH}_{2} \mathrm{CH}_{2} \mathrm{P}\right), 24.06,22.56\left(\mathrm{~d}, J=51.0 \mathrm{~Hz}, \mathrm{CH}_{2} \mathrm{P}\right), 21.99$ (d, $\left.J=4.1 \mathrm{~Hz}, \mathrm{CH}_{2} \mathrm{CH}_{2} \mathrm{P}\right) .{ }^{31} \mathrm{P}$ NMR $\left(81 \mathrm{MHz}, \mathrm{CDCl}_{3}\right) \delta: 25.23$. ES-MS $m / z$ for $\mathrm{C}_{24} \mathrm{H}_{26} \mathrm{O}_{2} \mathrm{P}[\mathrm{M}]^{+}$: calcd. 377.2, found 377.2.

(10-Carboxydecyl)triphenylphosphonium bromide (2b). White powder $(5.88 \mathrm{~g}, 97 \%) .{ }^{1} \mathrm{H}$ NMR $\left(200 \mathrm{MHz}, \mathrm{CDCl}_{3}\right) \delta: 8.82$ (bs, 1H, $\left.\mathrm{COOH}\right), 7.80-7.60$ (m, 15H, ArH), 3.66-3.44 (m, 2H, $\left.\mathrm{CH}_{2} \mathrm{P}\right), 2.29$ $\left(\mathrm{t}, J=7.0 \mathrm{~Hz}, 2 \mathrm{H}, \mathrm{CH}_{2} \mathrm{COOH}\right), 1.69-1.38\left(\mathrm{~m}, 6 \mathrm{H}, \mathrm{CH}_{2}\right), 1.31-1.00\left(\mathrm{~m}, 10 \mathrm{H}, \mathrm{CH}_{2}\right) .{ }^{13} \mathrm{C} \mathrm{NMR}(50 \mathrm{MHz}$, $\left.\mathrm{CDCl}_{3}\right) \delta: 177.66(\mathrm{C}=\mathrm{O}), 135.10\left(\mathrm{~d}, J=2.8 \mathrm{~Hz}, \mathrm{PPh}_{3}\right.$ para), $133.52\left(\mathrm{~d}, J=9.9 \mathrm{~Hz}, \mathrm{PPh}_{3}\right.$ ortho), $130.53(\mathrm{~d}$, $J=12.5 \mathrm{~Hz}, \mathrm{PPh}_{3}$ meta), 118.09 (d, $J=85.9 \mathrm{~Hz}, \mathrm{PPh}_{3}$ ipso), $34.40,30.28\left(\mathrm{~d}, J=15.9 \mathrm{~Hz}, \mathrm{CH}_{2} \mathrm{CH}_{2} \mathrm{CH}_{2} \mathrm{P}\right)$, 28.99, 28.86, 28.84, 28.82, 28.73, 24.65, $22.56\left(\mathrm{~d}, J=50.7 \mathrm{~Hz}, \mathrm{CH}_{2} \mathrm{P}\right), 22.44\left(\mathrm{~d}, J=4.5 \mathrm{~Hz}, \mathrm{CH}_{2} \mathrm{CH}_{2} \mathrm{P}\right) .{ }^{31} \mathrm{P}$ NMR $\left(81 \mathrm{MHz}, \mathrm{CDCl}_{3}\right) \delta$ : 25.08. ES-MS $m / z$ for $\mathrm{C}_{29} \mathrm{H}_{36} \mathrm{O}_{2} \mathrm{P}[\mathrm{M}]^{+}$: calcd. 447.2, found 447.2.

\subsubsection{Synthesis of the Nitrophthalic Anhydrides 4a,b}

A mixture of 3- or 4-nitrophthalic acid $(10 \mathrm{~g}, 0.047 \mathrm{~mol})$ and acetic anhydride $(24 \mathrm{~mL})$ was stirred at reflux for $1 \mathrm{~h}$. After cooling, volatiles were evaporated (repeated addition of toluene and evaporation facilitated the procedure). The anhydride precipitated out of the residue upon addition of diethyl ether as sub-white powder (3-nitrophthalic anhydride $4 \mathbf{a}: 7.90 \mathrm{~g}(90 \%)$, 4-nitrophthalic anhydride $4 \mathbf{b}: 8.00 \mathrm{~g}$ $(88 \%))$. The anhydrides were used in the next step without characterization.

\subsubsection{Synthesis of the Nitrophthalimides $5 \mathrm{a}, \mathrm{b}$}

A mixture of 3-nitrophthalic anhydride $(7 \mathrm{~g}, 36.25 \mathrm{mmol})$, sec-butylamine $(5.3 \mathrm{~g}, 72.50 \mathrm{mmol})$ and acetic acid $(60 \mathrm{~mL})$ was refluxed for $18 \mathrm{~h}$. After cooling, volatiles were evaporated and dichloromethane $(200 \mathrm{~mL})$ was added. The solution was washed with aq. $\mathrm{NaHCO}_{3}(2 \times 60 \mathrm{~mL})$ and water $(2 \times 60 \mathrm{~mL})$, dried $\left(\mathrm{Na}_{2} \mathrm{SO}_{4}\right)$ and the solvent evaporated, affording the desired phthalimides.

2-(sec-Butyl)-4-nitroisoindoline-1,3-dione (5a). From 3-nitrophthalic anhydride 4a. Beige solid, $7.92 \mathrm{~g}$ (88\%). ${ }^{1} \mathrm{H}$ NMR $\left(200 \mathrm{MHz}, \mathrm{CDCl}_{3}\right)$ 8: 8.10-8.03 (m, 2H, H-5, H-7), 7.94-7.85 (m, 1H, H-6), 4.35-4.17 (m, $1 \mathrm{H}, \mathrm{NCH}), 2.13-1.67\left(\mathrm{~m}, 2 \mathrm{H}, \mathrm{CH}_{2}\right), 1.45\left(\mathrm{~d}, J=7.0 \mathrm{~Hz}, 3 \mathrm{H}, \mathrm{CHCH}_{3}\right), 0.86\left(\mathrm{t}, J=7.4 \mathrm{~Hz}, 3 \mathrm{H}, \mathrm{CH}_{3}\right) .{ }^{13} \mathrm{C}$ NMR $\left(50 \mathrm{MHz}, \mathrm{CDCl}_{3}\right) \delta: 165.98,163.08,144.91,135.34,133.85,128.86,126.82,123.33,49.91,26.58,18.10$, 11.20. ES-MS $m / z$ for $\mathrm{C}_{12} \mathrm{H}_{12} \mathrm{~N}_{2} \mathrm{O}_{4}[\mathrm{M}]^{+}$: calcd. 248.0, found 248.0. ES-HRMS $m / z$ for $\mathrm{C}_{12} \mathrm{H}_{12} \mathrm{~N}_{2} \mathrm{NaO}_{4}$ $[\mathrm{M}+\mathrm{Na}]^{+}$: calcd. 248.0797, found 248.0801. 
2-(sec-Butyl)-5-nitroisoindoline-1,3-dione (5b). From 3-nitrophthalic anhydride $\mathbf{4 b}$. Beige solid, $7.74 \mathrm{~g}$ (86\%). ${ }^{1} \mathrm{H}$ NMR $\left(200 \mathrm{MHz}, \mathrm{CDCl}_{3}\right) \delta: 8.59$ (s, 1H, H-4), 8.57 (d, J = 7.5 Hz, 1H, H-6), 8.00 (d, J = 7.5 Hz, $1 \mathrm{H}, \mathrm{H}-7), 4.38-4.14(\mathrm{~m}, 1 \mathrm{H}, \mathrm{NCH}), 2.15-1.66\left(\mathrm{~m}, 2 \mathrm{H}, \mathrm{CH}_{2}\right), 1.46\left(\mathrm{~d}, \mathrm{~J}=7.0 \mathrm{~Hz}, 3 \mathrm{H}, \mathrm{CHCH}_{3}\right), 0.85(\mathrm{t}$, $\left.J=7.4 \mathrm{~Hz}, 3 \mathrm{H}, \mathrm{CH}_{3}\right) .{ }^{13} \mathrm{C} \mathrm{NMR}\left(50 \mathrm{MHz}, \mathrm{CDCl}_{3}\right) \delta: 166.38,166.07,151.62,136.35,133.28,129.14$, $124.28,118.43,49.92,26.69,18.19,11.20$. ES-HRMS $m / z$ for $\mathrm{C}_{12} \mathrm{H}_{12} \mathrm{~N}_{2} \mathrm{NaO}_{4}[\mathrm{M}+\mathrm{Na}]^{+}$: calcd. 248.0797, found 248.0803 .

\subsubsection{Synthesis of Aminophthalimides 6a,b}

A stirred solution of the nitrophthalimide $(1.83 \mathrm{~g}, 7.37 \mathrm{mmol})$ in methanol $(30 \mathrm{~mL})$ was degassed (Ar) for $30 \mathrm{~min} .10 \% \mathrm{Pd} / \mathrm{C}(200 \mathrm{mg})$ was added, then bubbled with $\mathrm{H}_{2}$ for a while and the mixture was stirred under an $\mathrm{H}_{2}$ atmosphere ( $20 \mathrm{bar}$ ) for $18 \mathrm{~h}$. The mixture was filtered through celite, washed with methanol, and the filtrate was concentrated, leaving the corresponding aminophthalimide.

4-Amino-2-(sec-butyl)isoindoline-1,3-dione (6a). From 4-nitrophthalimide 5a. Yellow solid, $1.50 \mathrm{~g}$ (93\%). ${ }^{1} \mathrm{H}$ NMR $\left(200 \mathrm{MHz}, \mathrm{CDCl}_{3}\right) \delta: 7.36(\mathrm{dd}, J=8.3,7.1 \mathrm{~Hz}, 1 \mathrm{H}, \mathrm{H}-6), 7.08(\mathrm{~d}, J=7.1 \mathrm{~Hz}, 1 \mathrm{H}, \mathrm{H}-5)$, $6.87(\mathrm{~d}, J=8.3 \mathrm{~Hz}, 1 \mathrm{H}, \mathrm{H}-7), 5.41\left(\mathrm{bs}, 2 \mathrm{H}, \mathrm{NH}_{2}\right), 4.28-4.01(\mathrm{~m}, 1 \mathrm{H}, \mathrm{NCH}), 2.17-1.64\left(\mathrm{~m}, 2 \mathrm{H}, \mathrm{CH}_{2}\right), 1.45$ $\left(\mathrm{d}, J=7.0 \mathrm{~Hz}, 3 \mathrm{H}, \mathrm{CHCH}_{3}\right), 0.87\left(\mathrm{t}, J=7.4 \mathrm{~Hz}, 3 \mathrm{H}, \mathrm{CH}_{3}\right) .{ }^{13} \mathrm{C} \mathrm{NMR}\left(50 \mathrm{MHz}, \mathrm{CDCl}_{3}\right) \delta: 170.51,168.84$, $145.27,134.82,132.52,120.88,112.10,110.92,48.48,26.81,18.40,11.23$. ES-HRMS $m / z$ for $\mathrm{C}_{12} \mathrm{H}_{14} \mathrm{~N}_{2} \mathrm{NaO}_{2}$ $[\mathrm{M}+\mathrm{Na}]^{+}$: calcd. 241.0947, found 241.0948.

5-Amino-2-(sec-butyl)isoindoline-1,3-dione (6b). From 5-nitrophthalimide 5b. Yellow solid, $1.51 \mathrm{~g}$ (94\%). ${ }^{1} \mathrm{H}$ NMR (200 MHz, DMSO- $\left.d_{6}\right) \delta: 7.45$ (d, $\left.J=8.1 \mathrm{~Hz}, 1 \mathrm{H}, \mathrm{H}-7\right), 6.89$ (s, 1H, H-4), 6.78 (d, $J=7.9 \mathrm{~Hz}, 1 \mathrm{H}, \mathrm{H}-6), 6.45\left(\mathrm{~s}, 2 \mathrm{H}, \mathrm{NH}_{2}\right), 4.09-3.95\left(\mathrm{~m}, 1 \mathrm{H}, \mathrm{CHCH}_{2}\right), 1.98-1.58\left(\mathrm{~m}, 2 \mathrm{H}, \mathrm{CH}_{2}\right), 1.34(\mathrm{~d}$, $\left.J=6.8 \mathrm{~Hz}, 3 \mathrm{H}, \mathrm{CHCH}_{3}\right), 0.76\left(\mathrm{t}, J=7.2 \mathrm{~Hz}, 3 \mathrm{H}, \mathrm{CH}_{3}\right) .{ }^{13} \mathrm{C} \mathrm{NMR}\left(50 \mathrm{MHz}, \mathrm{DMSO}-d_{6}\right): 168.55,168.27$, $155.02,134.29,124.81,116.65,116.49,106.83,47.77,26.45,18.45,11.22$. ES-HRMS $m / z$ for $\mathrm{C}_{12} \mathrm{H}_{14} \mathrm{~N}_{2} \mathrm{NaO}_{2}$ $[\mathrm{M}+\mathrm{Na}]^{+}$: calcd. 241.0947, found 241.0948.

\subsubsection{General Procedure for the Acylation of Phthalimides}

A solution of the carboxylic acid $(11 \mathrm{mmol})$ in oxalyl chloride $(10 \mathrm{~mL})$ was stirred for $5 \mathrm{~h}$ under Ar. Then, the volatiles were evaporated to dryness under reduced pressure at room temperature. The residue was dissolved in dry dichloromethane $(8 \mathrm{~mL})$ and added dropwise to a cooled $\left(0^{\circ} \mathrm{C}\right)$ solution of the aminophthalimide $(2.18 \mathrm{~g}, 10 \mathrm{mmol})$ and pyridine $(1.61 \mathrm{~mL}, 20 \mathrm{mmol})$ in dichloromethane $(24 \mathrm{~mL})$ under Ar. The resulting mixture was stirred at r.t. for $18 \mathrm{~h}$. Water $(100 \mathrm{~mL})$ was added, the layers were separated and the aqueous was washed with dichloromethane $(2 \times 40 \mathrm{~mL})$. The combined organic layers were dried $\left(\mathrm{Na}_{2} \mathrm{SO}_{4}\right)$, solvent was evaporated and the residue was subjected to column chromatography, affording the corresponding acylated phthalimide.

6-Bromo-N-(2-(sec-butyl)-1,3-dioxoisoindolin-4-yl)hexanamide (8a). From 6-bromohexanoic acid and 4-aminophthalimide 6a. Chromatography with EtOAc/petroleum ether 8:1 to 4:1. Brownish oil (2.8 g, 71\%). ${ }^{1} \mathrm{H}$ NMR (200 MHz, $\left.\mathrm{CDCl}_{3}\right) \delta: 9.60(\mathrm{bs}, 1 \mathrm{H}, \mathrm{NH}), 8.73(\mathrm{~d}, J=8.4 \mathrm{~Hz}, 1 \mathrm{H}, \mathrm{H}-5), 7.63(\mathrm{t}, J=7.9 \mathrm{~Hz}$, 1H, H-6), $7.45(\mathrm{~d}, J=7.2 \mathrm{~Hz}, 1 \mathrm{H}, \mathrm{H}-7), 4.28-4.09(\mathrm{~m}, 1 \mathrm{H}, \mathrm{CH}), 3.41\left(\mathrm{t}, J=6.7 \mathrm{~Hz}, 2 \mathrm{H}, \mathrm{BrCH}_{2}\right), 2.47(\mathrm{t}$, $\left.J=7.4 \mathrm{~Hz}, 2 \mathrm{H}, \mathrm{CH}_{2} \mathrm{CO}\right), 2.12-1.65(\mathrm{~m}, 6 \mathrm{H}), 1.60-1.42(\mathrm{~m}, 5 \mathrm{H}), 0.86\left(\mathrm{t}, J=7.4 \mathrm{~Hz}, 3 \mathrm{H}, \mathrm{CH}_{2} \mathrm{CH}_{3}\right) .{ }^{13} \mathrm{C}$ NMR $\left(50 \mathrm{MHz} \mathrm{CDCl}_{3}\right) \delta: 172.01,170.71,168.05,137.24,135.78,131.37,124.61,117.83,115.55,49.19$, $37.71,33.63,32.45,27.72,26.90,24.40,18.54,11.39$. ES-HRMS $m / z$ for $\mathrm{C}_{18} \mathrm{H}_{22} \mathrm{BrN}_{2} \mathrm{O}_{3}[\mathrm{M}-\mathrm{H}]^{-}$: calcd. 393.0819, found 393.0821.

11-Bromo-N-(2-(sec-butyl)-1,3-dioxoisoindolin-4-yl)undecanamide (8b). From 11-bromoundecanoic acid and 4-aminophthalimide 6a. Chromatography with EtOAc/petroleum ether 8:1 to 4:1. Brownish oil (3.3 g, 70\%). ${ }^{1} \mathrm{H}$ NMR (200 MHz, $\left.\mathrm{CDCl}_{3}\right)$ 8: $9.58(\mathrm{bs}, 1 \mathrm{H}, \mathrm{NH}), 8.74(\mathrm{~d}, J=8.4 \mathrm{~Hz}, 1 \mathrm{H}, \mathrm{H}-5), 7.62(\mathrm{t}$, $J=7.9 \mathrm{~Hz}, 1 \mathrm{H}, \mathrm{H}-6), 7.44(\mathrm{~d}, J=7.3 \mathrm{~Hz}, 1 \mathrm{H}, \mathrm{H}-7), 4.27-4.10(\mathrm{~m}, 1 \mathrm{H}, \mathrm{CH}), 3.37\left(\mathrm{t}, J=6.8 \mathrm{~Hz}, 2 \mathrm{H}, \mathrm{BrCH}_{2}\right)$, $2.44\left(\mathrm{t}, J=7.5 \mathrm{~Hz}, 2 \mathrm{H}, \mathrm{CH}_{2} \mathrm{CO}\right), 2.08-1.65(\mathrm{~m}, 6 \mathrm{H}), 1.45-1.19(\mathrm{~m}, 15 \mathrm{H}), 0.86\left(\mathrm{t}, J=7.4 \mathrm{~Hz}, 3 \mathrm{H}, \mathrm{CH}_{2} \mathrm{CH}_{3}\right)$. ${ }^{13} \mathrm{C}$ NMR $\left(50 \mathrm{MHz}, \mathrm{CDCl}_{3}\right) \delta: 172.49,170.69,167.93,137.40,135.71,131.43,124.64,117.70,115.57,77.16$, 
$49.20,38.06,34.08,32.87,29.41,29.38,29.29,29.19,28.78,28.20,26.93,25.33,18.50,11.36$. ES-HRMS $\mathrm{m} / \mathrm{z}$ for $\mathrm{C}_{23} \mathrm{H}_{32} \mathrm{BrN}_{2} \mathrm{O}_{3}[\mathrm{M}-\mathrm{H}]^{-}$: calcd. 463.1602 , found 463.1613 .

6-Bromo-N-(2-(sec-butyl)-1,3-dioxoisoindolin-4-yl)hexanamide (9a). From 6-bromohexanoic acid and 5-aminophthalimide 6 b. Chromatography with methanol/DCM 0\% to 5\%. Brownish oil $(2.7 \mathrm{~g}, 40 \%$, mixture with $10 \mathrm{~mol} \%$ of the corresponding chloride). ${ }^{1} \mathrm{H}$ NMR $\left(200 \mathrm{MHz}, \mathrm{CDCl}_{3}\right) \delta: 8.01(\mathrm{dd}, J=8.1$, $1.8 \mathrm{~Hz}, 1 \mathrm{H}, \mathrm{H}-6), 7.96(\mathrm{~d}, J=1.5 \mathrm{~Hz}, 1 \mathrm{H}, \mathrm{H}-4), 7.94(\mathrm{bs}, 1 \mathrm{H}, \mathrm{NH}), 7.75(\mathrm{~d}, J=8.1 \mathrm{~Hz}, 1 \mathrm{H}, \mathrm{H}-7), 4.32-4.14$ $(\mathrm{m}, 1 \mathrm{H}, \mathrm{CH}), 3.42\left(\mathrm{t}, J=6.6 \mathrm{~Hz}, 2 \mathrm{H}, \mathrm{BrCH}_{2}\right), 2.47\left(\mathrm{t}, J=7.3 \mathrm{~Hz}, 2 \mathrm{H}, \mathrm{CH}_{2} \mathrm{CO}\right), 2.15-1.71(\mathrm{~m}, 6 \mathrm{H}), 1.61-1.44$ $(\mathrm{m}, 5 \mathrm{H}), 0.86\left(\mathrm{t}, J=7.4 \mathrm{~Hz}, 3 \mathrm{H}, \mathrm{CH}_{2} \mathrm{CH}_{3}\right) .{ }^{13} \mathrm{C} \mathrm{NMR}\left(50 \mathrm{MHz}, \mathrm{CDCl}_{3}\right) \delta: 172.38,168.10,168.02,143.71$, $132.97,126.00,123.82,123.75,113.82,48.85,37.00,33.33,32.06,27.39,26.57,24.29,18.11,11.04$. ES-HRMS $m / z$ for $\mathrm{C}_{18} \mathrm{H}_{22} \mathrm{BrN}_{2} \mathrm{O}_{3}[\mathrm{M}-\mathrm{H}]^{-}$: calcd. 393.0819, found 393.0811.

11-Bromo- $N$-(2-(sec-butyl)-1,3-dioxoisoindolin-5-yl)undecanamide (9b). From 11-bromoundecanoic acid and 5-aminophthalimide $\mathbf{6 b}$. Chromatography with methanol/DCM $0 \%$ to $5 \%$. Brownish oil (2.7 g, 58\%). ${ }^{1} \mathrm{H}$ NMR $\left(200 \mathrm{MHz}, \mathrm{CDCl}_{3}\right) \delta: 8.25$ (bs, $\left.1 \mathrm{H}, \mathrm{NH}\right), 8.09$ (d, $\left.J=8.2 \mathrm{~Hz}, 1 \mathrm{H}, \mathrm{H}-6\right), 7.97(\mathrm{~s}$, $1 \mathrm{H}, \mathrm{H}-4), 7.74(\mathrm{~d}, J=8.1 \mathrm{~Hz}, 1 \mathrm{H}, \mathrm{H}-7), 4.32-4.13(\mathrm{~m}, 1 \mathrm{H}, \mathrm{CH}), 3.38\left(\mathrm{t}, J=6.7 \mathrm{~Hz}, 2 \mathrm{H}, \mathrm{BrCH}_{2}\right), 2.45(\mathrm{t}$, $\left.J=6.8 \mathrm{~Hz}, 2 \mathrm{H}, \mathrm{CH}_{2} \mathrm{CO}\right), 2.08-1.65(\mathrm{~m}, 6 \mathrm{H}), 1.47-1.19(\mathrm{~m}, 15 \mathrm{H}), 0.86\left(\mathrm{t}, J=7.3 \mathrm{~Hz}, 3 \mathrm{H}, \mathrm{CH}_{2} \mathrm{CH}_{3}\right) .{ }^{13} \mathrm{C}$ NMR $\left(50 \mathrm{MHz}, \mathrm{CDCl}_{3}\right) \delta: 172.16,168.60,168.29,143.79,133.45,126.43,124.46,123.84,113.86,49.25$, 37.91, 34.25, 32.86, 29.45, 29.42 (2C), 29.32, 28.80, 28.21, 26.96, 25.49, 18.54, 11.43. ES-HRMS $m / z$ for $\mathrm{C}_{23} \mathrm{H}_{32} \mathrm{BrN}_{2} \mathrm{O}_{3}[\mathrm{M}-\mathrm{H}]^{-}$: calcd. 463.1602, found 463.1610 .

\subsubsection{General Procedure for the Synthesis of Phosphonium Phthalimides}

A solution of the bromide $(1 \mathrm{mmol})$ and the phosphine $(2 \mathrm{mmol})$ in dry acetonitrile $(5 \mathrm{~mL})$ was refluxed under Ar for 3 days. After cooling, the solvent was evaporated and the residue was subjected to column chromatography, yielding the corresponding phosphonium cation.

(6-((2-(sec-Butyl)-1,3-dioxoisoindolin-4-yl)amino)-6-oxohexyl)triphenylphosphonium bromide (10a). From bromide 8a and triphenylphosphine. Chromatography with methanol/DCM 3 to $10 \%$. White solid (335 mg, 51\%). ${ }^{1} \mathrm{H}$ NMR (200 MHz, $\left.\mathrm{CDCl}_{3}\right) \delta$ : $9.53(\mathrm{bs}, 1 \mathrm{H}, \mathrm{NH}), 8.62(\mathrm{~d}, J=8.4 \mathrm{~Hz}, \mathrm{H}-5), 7.85-7.60$ $(\mathrm{m}, 15 \mathrm{H}, \mathrm{ArH}), 7.55(\mathrm{t}, J=8.0 \mathrm{~Hz}, 1 \mathrm{H}, \mathrm{H}-6), 7.39(\mathrm{~d}, J=7.2 \mathrm{~Hz}, 1 \mathrm{H}, \mathrm{H}-7), 4.23-4.05(\mathrm{~m}, 1 \mathrm{H}, \mathrm{CH})$, 3.80-3.65 (m, 2H, $\left.\mathrm{PCH}_{2}\right), 2.40\left(\mathrm{t}, J=6.1 \mathrm{~Hz}, 2 \mathrm{H}, \mathrm{CH}_{2} \mathrm{CO}\right), 2.07-1.60(\mathrm{~m}, 8 \mathrm{H}), 1.39(\mathrm{~d}, J=6.9 \mathrm{~Hz}, 3 \mathrm{H}$, $\left.\mathrm{CH}_{3}\right), 0.81\left(\mathrm{t}, J=7.3 \mathrm{~Hz}, 3 \mathrm{H}, \mathrm{CH}_{2} \mathrm{CH}_{3}\right) .{ }^{13} \mathrm{C} \mathrm{NMR}\left(50 \mathrm{MHz}, \mathrm{CDCl}_{3}\right) \delta: 171.16,169.35,167.10,136.11$, 134.80, $134.34\left(\mathrm{~d}, J=2.7 \mathrm{~Hz}, \mathrm{PPh}_{3}\right.$ para), $132.74\left(\mathrm{~d}, J=10.0 \mathrm{~Hz}, \mathrm{PPh}_{3}\right.$ ortho), $130.45,129.76$ (d, $J=12.5 \mathrm{~Hz}$, $\mathrm{PPh}_{3}$ meta), 123.89, 117.21 (d, $J=86.0 \mathrm{~Hz}, \mathrm{PPh}_{3}$ ipso), 116.90, 114.88, 48.90, 36.26, 28.82 (d, $J=16.0 \mathrm{~Hz}$, $\left.\mathrm{CH}_{2} \mathrm{CH}_{2} \mathrm{CH}_{2} \mathrm{P}\right), 25.94,23.52,21.44\left(\mathrm{~d}, J=50.0 \mathrm{~Hz}, \mathrm{CH}_{2} \mathrm{P}\right), 21.40\left(\mathrm{~d}, J=4.0 \mathrm{~Hz}, \mathrm{CH}_{2} \mathrm{CH}_{2} \mathrm{P}\right), 17.60,10.49$. ${ }^{31} \mathrm{P}$ NMR $\left(81 \mathrm{MHz}, \mathrm{CDCl}_{3}\right) \delta$ : 24.89. ES-HRMS $\mathrm{m} / z$ for $\mathrm{C}_{36} \mathrm{H}_{38} \mathrm{~N}_{2} \mathrm{O}_{3} \mathrm{P}[\mathrm{M}]^{+}$: calcd. 577.2615, found 577.2608.

(6-((2-(sec-Butyl)-1,3-dioxoisoindolin-4-yl)amino)-6-oxohexyl)tri- $p$-tolylphosphonium bromide (10b). From bromide 8a and tri(p-tolyl)phosphine. Chromatography with methanol/DCM 5\% to $20 \%$. White solid (371 mg, 53\%). ${ }^{1} \mathrm{H}$ NMR (200 MHz, $\left.\mathrm{CDCl}_{3}\right) \delta$ : 9.56 (bs, $\left.1 \mathrm{H}, \mathrm{NH}\right), 8.67(\mathrm{~d}, J=8.4 \mathrm{~Hz}, \mathrm{H}-5), 7.70-7.57$ (m, 7H, ArH), 7.50-7.43 (m, 7H, ArH, H-6, H-7), 4.26-4.08 (m, 1H, CH), 3.64-3.50 (m, 2H, $\left.\mathrm{PCH}_{2}\right)$, 2.46-2.34 (m, 11H, $\left.\mathrm{ArCH}_{3}, \mathrm{CH}_{2} \mathrm{CO}\right), 2.07-1.60(\mathrm{~m}, 8 \mathrm{H}), 1.43\left(\mathrm{~d}, J=6.9 \mathrm{~Hz}, 3 \mathrm{H}, \mathrm{CH}_{3}\right), 0.85(\mathrm{t}, J=7.4 \mathrm{~Hz}$, $\left.3 \mathrm{H}, \mathrm{CH}_{2} \mathrm{CH}_{3}\right) .{ }^{13} \mathrm{C}$ NMR $\left(50 \mathrm{MHz}, \mathrm{CDCl}_{3}\right) \delta: 171.88,170.24,167.92,146.15$ (d, $J=3.0 \mathrm{~Hz}, \mathrm{PAr}_{3}$ para), 136.94, 135.47, 133.34 (d, $J=10.3 \mathrm{~Hz}, \mathrm{PAr}_{3}$ ortho), 131.26, 131.09 (d, $J=12.9 \mathrm{~Hz}, \mathrm{PAr}_{3}$ meta), 124.57, $117.64,115.59,114.83$ (d, $J=88.7 \mathrm{~Hz}, \mathrm{PAr}_{3}$ ipso), 48.98, 37.03, $29.63\left(\mathrm{~d}, J=16.6 \mathrm{~Hz}, \mathrm{CH}_{2} \mathrm{CH}_{2} \mathrm{CH}_{2} \mathrm{P}\right), 26.70$, 24.35, $22.66\left(\mathrm{~d}, J=53.0 \mathrm{~Hz}, \mathrm{CH}_{2} \mathrm{P}\right), 22.18\left(\mathrm{~d}, J=4.5 \mathrm{~Hz}, \mathrm{CH}_{2} \mathrm{CH}_{2} \mathrm{P}\right), 21.79\left(\mathrm{~d}, J=1.2 \mathrm{~Hz}, \mathrm{ArCH}_{3}\right), 18.36$, 11.23. ${ }^{31} \mathrm{P}$ NMR $\left(81 \mathrm{MHz}, \mathrm{CDCl}_{3}\right) \delta$ : 23.97. ES-HRMS $m / z$ for $\mathrm{C}_{39} \mathrm{H}_{44} \mathrm{~N}_{2} \mathrm{O}_{3} \mathrm{P}[\mathrm{M}]^{+}$: calcd. 619.3084, found 619.3080 .

(11-((2-(sec-Butyl)-1,3-dioxoisoindolin-4-yl)amino)-11-oxoundecyl)triphenylphosphonium bromide (10c). From bromide $\mathbf{8 b}$ and triphenylphosphine. Chromatography with methanol/DCM 5\% to 10\%. White solid (582 mg, 80\%). ${ }^{1} \mathrm{H}$ NMR (200 MHz, $\left.\mathrm{CDCl}_{3}\right) \delta$ : 9.55 (bs, $\left.1 \mathrm{H}, \mathrm{NH}\right), 8.71$ (d, J = 8.4 Hz, H-5), 
7.84-7.55 (m, 16H, ArH, H-6), $7.41(\mathrm{~d}, J=7.2 \mathrm{~Hz}, 1 \mathrm{H}, \mathrm{H}-7), 4.24-4.07$ (m, 1H, CH), 3.77-3.61 (m, 2H, $\left.\mathrm{PCH}_{2}\right), 2.39\left(\mathrm{t}, J=7.5 \mathrm{~Hz}, 2 \mathrm{H}, \mathrm{CH}_{2} \mathrm{CO}\right), 2.09-1.58(\mathrm{~m}, 6 \mathrm{H}), 1.41\left(\mathrm{~d}, J=6.9 \mathrm{~Hz}, 3 \mathrm{H}, \mathrm{CH}_{3}\right), 1.34-1.10$ $(\mathrm{m}, 12 \mathrm{H}), 0.83\left(\mathrm{t}, J=7.4 \mathrm{~Hz}, 3 \mathrm{H}, \mathrm{CH}_{2} \mathrm{CH}_{3}\right) .{ }^{13} \mathrm{C} \mathrm{NMR}\left(50 \mathrm{MHz}, \mathrm{CDCl}_{3}\right) \delta: 172.55,170.70,168.12$, 137.42, 135.71, 135.08 (d, J = 3.0 Hz, $\mathrm{PPh}_{3}$ para), 133.79 (d, $J=9.9 \mathrm{~Hz}, \mathrm{PPh}_{3}$ ortho), 131.48, 130.58 (d, $J=12.6 \mathrm{~Hz}, \mathrm{PPh}_{3}$ meta), $124.69,118.25$ (d, $J=85.9 \mathrm{~Hz}, \mathrm{PPh}_{3}$ ipso), 117.72, 115.63, 49.24, 38.06, 30.49 (d, $\left.J=15.5 \mathrm{~Hz}, \mathrm{CH}_{2} \mathrm{CH}_{2} \mathrm{CH}_{2} \mathrm{P}\right), 29.37,29.27(2 \mathrm{C}), 29.18(2 \mathrm{C}), 26.95,25.32,22.87\left(\mathrm{~d}, J=49.6 \mathrm{~Hz}, \mathrm{CH}_{2} \mathrm{P}\right)$, $22.75\left(\mathrm{~d}, J=4.4 \mathrm{~Hz}, \mathrm{CH}_{2} \mathrm{CH}_{2} \mathrm{P}\right), 18.52,11.37 .{ }^{31} \mathrm{P} \mathrm{NMR}\left(81 \mathrm{MHz}, \mathrm{CDCl}_{3}\right) \delta: 25.31$. ES-HRMS $\mathrm{m} / \mathrm{z}$ for $\mathrm{C}_{41} \mathrm{H}_{48} \mathrm{~N}_{2} \mathrm{O}_{3} \mathrm{P}[\mathrm{M}]^{+}$: calcd. 647.3397, found 647.3397.

(11-((2-(sec-Butyl)-1,3-dioxoisoindolin-4-yl)amino)-11-oxoundecyl)tri- $p$-tolylphosphonium bromide (10d). From bromide $8 \mathbf{b}$ and tri(p-tolyl)phosphine. Chromatography with methanol/DCM $5 \%$ to $10 \%$. White solid (485 mg, 63\%). ${ }^{1} \mathrm{H}$ NMR $\left(200 \mathrm{MHz}, \mathrm{CDCl}_{3}\right) \delta: 9.57(\mathrm{bs}, 1 \mathrm{H}, \mathrm{NH}), 8.73(\mathrm{~d}, J=8.4 \mathrm{~Hz}$, H-5), 7.68-7.42 (m, 14H, ArH, H-6, H-7), 4.26-4.09 (m, 1H, CH), 3.57-3.42 (m, 2H, PCH $), 2.44$ (s, 9H, $\left.\mathrm{ArCH}_{3}\right), 2.39\left(\mathrm{t}, J=7.9 \mathrm{~Hz}, 2 \mathrm{H}, \mathrm{CH}_{2} \mathrm{CO}\right), 2.07-1.57(\mathrm{~m}, 6 \mathrm{H}), 1.43\left(\mathrm{~d}, J=7.0 \mathrm{~Hz}, 3 \mathrm{H}, \mathrm{CH}_{3}\right), 1.36-1.13(\mathrm{~m}$, $12 \mathrm{H}), 0.88\left(\mathrm{t}, J=7.4 \mathrm{~Hz}, 3 \mathrm{H}, \mathrm{CH}_{2} \mathrm{CH}_{3}\right) .{ }^{13} \mathrm{C} \mathrm{NMR}\left(50 \mathrm{MHz}, \mathrm{CDCl}_{3}\right) \delta: 172.55,170.67,168.10,146.25(\mathrm{~d}$, $J=3.0 \mathrm{~Hz}, \mathrm{PAr}_{3}$ para), 137.36, 135.70, 133.53 (d, $J=10.3 \mathrm{~Hz}, \mathrm{PAr}_{3}$ ortho), 131.41, 131.21 (d, $J=12.9 \mathrm{~Hz}$, $\mathrm{PAr}_{3}$ meta), 124.64, 117.71, 115.56, 115.19 (d, $J=88.6 \mathrm{~Hz}, \mathrm{PAr}_{3}$ ipso), 49.20, 38.04, 30.55 (d, J = $15.6 \mathrm{~Hz}$, $\left.\mathrm{CH}_{2} \mathrm{CH}_{2} \mathrm{CH}_{2} \mathrm{P}\right), 29.76,29.38,29.27,29.22,29.17,26.91,25.30,23.06\left(\mathrm{~d}, J=51.1 \mathrm{~Hz}, \mathrm{CH}_{2} \mathrm{P}\right), 22.67(\mathrm{~d}$, $\left.J=4.3 \mathrm{~Hz}, \mathrm{CH}_{2} \mathrm{CH}_{2} \mathrm{P}\right), 21.94\left(\mathrm{~d}, J=1.2 \mathrm{~Hz}, \mathrm{ArCH}_{3}\right) 18.52,11.37 .{ }^{31} \mathrm{P} \mathrm{NMR}\left(81 \mathrm{MHz}, \mathrm{CDCl}_{3}\right) \delta: 24.13$. ES-MS m/z for $\mathrm{C}_{44} \mathrm{H}_{54} \mathrm{~N}_{2} \mathrm{O}_{3} \mathrm{P}[\mathrm{M}]^{+}$: calcd. 689.3867, found 689.3869 .

(11-((2-(sec-Butyl)-1,3-dioxoisoindolin-4-yl)amino)-11-oxoundecyl)tricyclohexylphosphonium bromide (10e). From bromide $\mathbf{8 b}$ and tricyclohexylphosphine. Chromatography with methanol/DCM 5\% to $10 \%$. White solid $(723 \mathrm{mg}, 97 \%) .{ }^{1} \mathrm{H}$ NMR $\left(200 \mathrm{MHz}, \mathrm{CDCl}_{3}\right) \delta: 9.51(\mathrm{bs}, 1 \mathrm{H}, \mathrm{NH}), 8.65(\mathrm{~d}, J=8.3 \mathrm{~Hz}$, $\mathrm{H}-5), 7.55(\mathrm{t}, J=8.1 \mathrm{~Hz}, 1 \mathrm{H}, \mathrm{H}-6), 7.36(\mathrm{~d}, J=7.2 \mathrm{~Hz}, 1 \mathrm{H}, \mathrm{H}-7), 4.20-4.02(\mathrm{~m}, 1 \mathrm{H}, \mathrm{CH}), 2.67-2.22(\mathrm{~m}$, $\left.7 \mathrm{H}, \mathrm{PCH}, \mathrm{COCH}_{2}\right), 1.96-1.15(\mathrm{~m}, 51 \mathrm{H}, \mathrm{CH}), 0.78\left(\mathrm{t}, J=7.3 \mathrm{~Hz}, 3 \mathrm{H}, \mathrm{CH}_{2} \mathrm{CH}_{3}\right) .{ }^{13} \mathrm{C} \mathrm{NMR}(50 \mathrm{MHz}$, $\left.\mathrm{CDCl}_{3}\right) \delta:$ 172.07, 170.18, 167.63, 136.92, 135.27, 130.98, 124.19, 117.24, 115.14, 48.73, 37.59, 30.91 (d, $\left.J=13.6 \mathrm{~Hz}, \mathrm{CH}_{2} \mathrm{CH}_{2} \mathrm{CH}_{2} \mathrm{P}\right), 29.46\left(\mathrm{~d}, J=40.3 \mathrm{~Hz}, \mathrm{PCy}_{3}-\mathrm{C} 1\right), 28.91,28.88,28.86,28.73,28.61,26.87(\mathrm{~d}$, $\left.J=3.5 \mathrm{~Hz}, \mathrm{PCy}_{3}-\mathrm{C} 2\right), 26.46,26.08\left(\mathrm{~d}, J=11.8 \mathrm{~Hz}, \mathrm{PCy}_{3}-\mathrm{C} 3\right), 25.08,24.86,22.44\left(\mathrm{~d}, J=5.2 \mathrm{~Hz}, \mathrm{CH}_{2} \mathrm{CH}_{2} \mathrm{P}\right)$, 18.08, $15.45\left(\mathrm{~d}, J=43.0 \mathrm{~Hz}, \mathrm{CH}_{2} \mathrm{P}\right), 10.94 .{ }^{31} \mathrm{P} \mathrm{NMR}\left(81 \mathrm{MHz}, \mathrm{CDCl}_{3}\right) \delta: 32.62$. ES-HRMS $\mathrm{m} / z$ for $\mathrm{C}_{41} \mathrm{H}_{66} \mathrm{~N}_{2} \mathrm{O}_{3} \mathrm{P}[\mathrm{M}]^{+}$: calcd. 665.4806, found 665.4804.

(6-((2-(sec-Butyl)-1,3-dioxoisoindolin-5-yl)amino)-6-oxohexyl)tri-p-tolylphosphonium bromide (11a). From bromide $9 \mathrm{a}$ and tri( $p$-tolyl)phosphine. Chromatography with methanol/DCM 3 to $10 \%$. White solid (504 mg, 72\%). ${ }^{1} \mathrm{H}$ NMR (200 MHz, CDCl $) \delta: 10.98$ (bs, 1H, NH), 8.59 (s, 1H, H-4), 8.13 (d, $J=7.9 \mathrm{~Hz}, \mathrm{H}-6), 7.67-7.43(\mathrm{~m}, 13 \mathrm{H}, \mathrm{ArH}, \mathrm{H}-7), 4.29-4.10(\mathrm{~m}, 1 \mathrm{H}, \mathrm{CH}), 3.45-3.26\left(\mathrm{~m}, 2 \mathrm{H}, \mathrm{PCH}_{2}\right), 2.65$ $\left(\mathrm{t}, J=7.2 \mathrm{~Hz}, \mathrm{CH}_{2} \mathrm{CO}\right), 2.46\left(\mathrm{~s}, 9 \mathrm{H}, \mathrm{ArCH}_{3}\right), 2.06-1.70(\mathrm{~m}, 8 \mathrm{H}), 1.43\left(\mathrm{~d}, J=6.9 \mathrm{~Hz}, 3 \mathrm{H}, \mathrm{CH}_{3}\right), 0.84(\mathrm{t}$, $\left.J=7.4 \mathrm{~Hz}, 3 \mathrm{H}, \mathrm{CH}_{2} \mathrm{CH}_{3}\right) .{ }^{13} \mathrm{C} \mathrm{NMR}\left(50 \mathrm{MHz}, \mathrm{CDCl}_{3}\right) \delta: 173.10,168.45,168.28,146.26\left(\mathrm{~d}, J=3.0 \mathrm{~Hz}, \mathrm{PAr}_{3}\right.$ para), 145.00, 133.09 (d, $J=10.3 \mathrm{~Hz}, \mathrm{PAr}_{3}$ ortho), 132.66, 131.03 (d, $J=12.9 \mathrm{~Hz}, \mathrm{PAr}_{3}$ meta), 125.20, 124.14, 123.14, 114.49 (d, $J=88.9 \mathrm{~Hz}, \mathrm{PAr}_{3}$ ipso), 113.83, 48.47, 36.72, 29.70 (d, $\left.J=15.9 \mathrm{~Hz}, \mathrm{CH}_{2} \mathrm{CH}_{2} \mathrm{CH}_{2} \mathrm{P}\right), 26.61$, 24.32, $22.87\left(\mathrm{~d}, J=52.5 \mathrm{~Hz}, \mathrm{CH}_{2} \mathrm{P}\right), 21.64\left(\mathrm{~d}, J=1.2 \mathrm{~Hz}, \mathrm{ArCH}_{3}\right), 21.39\left(\mathrm{~d}, J=4.0 \mathrm{~Hz}, \mathrm{CH}_{2} \mathrm{CH}_{2} \mathrm{P}\right) 18.22$, 11.07. ${ }^{31} \mathrm{P}$ NMR (81 MHz, $\left.\mathrm{CDCl}_{3}\right) \delta$ : 22.24. ES-HRMS $m / z$ for $\mathrm{C}_{39} \mathrm{H}_{44} \mathrm{~N}_{2} \mathrm{O}_{3} \mathrm{P}[\mathrm{M}]^{+}$: calcd. 619.3084, found 619.3094 .

(6-((2-(sec-Butyl)-1,3-dioxoisoindolin-5-yl)amino)-6-oxohexyl)tricyclohexylphosphonium bromide (11b). From bromide 9a and tricyclohexylphosphine. Chromatography with methanol/DCM 3\% to $10 \%$. White solid (595 mg, 88\%, contaminated with $25 \mathrm{~mol} \%$ tricyclohexylphosphinoxide). ${ }^{1} \mathrm{H} \mathrm{NMR}$ $\left(400 \mathrm{MHz}, \mathrm{CDCl}_{3}\right) \delta: 10.87(\mathrm{bs}, 1 \mathrm{H}, \mathrm{NH}), 8.42(\mathrm{~d}, J=1.8 \mathrm{~Hz}, 1 \mathrm{H}, \mathrm{H}-4), 8.04(\mathrm{dd}, J=8.2,1.9 \mathrm{~Hz}, \mathrm{H}-6), 7.45$ $(\mathrm{d}, J=8.1 \mathrm{~Hz}, 1 \mathrm{H}, \mathrm{H}-7), 4.06-3.97(\mathrm{~m}, 1 \mathrm{H}, \mathrm{NCH}), 2.57\left(\mathrm{t}, J=7.3 \mathrm{~Hz}, \mathrm{CH}_{2} \mathrm{CO}\right), 2.48-2.39(\mathrm{~m}, 3 \mathrm{H}, \mathrm{PCH})$, 2.18-2.11 (m, 2H, $\left.\mathrm{PCH}_{2}\right), 1.97-1.05\left(\mathrm{~m}, 41 \mathrm{H}, \mathrm{CH}, \mathrm{O}=\mathrm{CCy}_{3}{ }^{*}\right), 0.67\left(\mathrm{t}, J=7.4 \mathrm{~Hz}, 3 \mathrm{H}, \mathrm{CH}_{2} \mathrm{CH}_{3}\right) .{ }^{13} \mathrm{C}$ $\operatorname{NMR}\left(100 \mathrm{MHz}, \mathrm{CDCl}_{3}\right) \delta: 172.85,168.33,168.20,144.95,132.66,125.25,123.98,123.09,113.64,48.45$, $36.11,35.02 *\left(\mathrm{~d}, J=60.8 \mathrm{~Hz}, \mathrm{O}=\mathrm{PCy}_{3}-\mathrm{C} 1\right), 30.00\left(\mathrm{~d}, J=14.1 \mathrm{~Hz}, \mathrm{CH}_{2} \mathrm{CH}_{2} \mathrm{CH}_{2} \mathrm{P}\right), 29.64(\mathrm{~d}, J=40.4 \mathrm{~Hz}$, 
$\left.\mathrm{PCy}_{3}-\mathrm{C} 1\right), 26.90\left(\mathrm{~d}, J=3.8 \mathrm{~Hz}, \mathrm{PCy}_{3}-\mathrm{C} 2\right), 26.59^{*}\left(\mathrm{~d}, J=11.6 \mathrm{~Hz}, \mathrm{O}=\mathrm{PCy}_{3}-\mathrm{C} 3\right), 26.55,26.12(\mathrm{~d}, J=11.9 \mathrm{~Hz}$, $\left.\mathrm{PCy}_{3}-\mathrm{C} 3\right), 26.02^{*}\left(\mathrm{~d}, J=3.0 \mathrm{~Hz}, \mathrm{O}=\mathrm{PCy}_{3}-\mathrm{C} 2\right), 25.82^{*}\left(\mathrm{~d}, J=1.3 \mathrm{~Hz}, \mathrm{O}=\mathrm{PCy}_{3}-\mathrm{C} 4\right), 25.07(\mathrm{~d}, J=1.2 \mathrm{~Hz}$, $\left.\mathrm{PCy}_{3}-\mathrm{C} 4\right), 24.20$ (d, $J=1.0 \mathrm{~Hz}, \mathrm{CH}_{2} \mathrm{CH}_{2} \mathrm{CH}_{2} \mathrm{CH}_{2} \mathrm{P}$ ), 21.43 (d, $\left.J=4.7 \mathrm{~Hz}, \mathrm{CH}_{2} \mathrm{CH}_{2} \mathrm{P}\right), 18.13,15.48$ (d, $\left.J=42.8 \mathrm{~Hz}, \mathrm{CH}_{2} \mathrm{P}\right), 10.96 .{ }^{31} \mathrm{P} \mathrm{NMR}\left(81 \mathrm{MHz}, \mathrm{CDCl}_{3}\right) \delta: 33.64$. ES-HRMS $m / z$ for $\mathrm{C}_{36} \mathrm{H}_{56} \mathrm{~N}_{2} \mathrm{O}_{3} \mathrm{P}[\mathrm{M}]^{+}$: calcd. 595.4023, found 595.4032. *signals attributed to tricyclohexylphosphinoxide.

(11-((2-(sec-Butyl)-1,3-dioxoisoindolin-5-yl)amino)-11-oxoundecyl)tri- $p$-tolylphosphonium bromide (11c). From bromide $9 \mathbf{b}$ and tri( $p$-tolyl)phosphine. Chromatography with methanol/DCM 3 to $10 \%$. White solid (523 mg, 68\%). ${ }^{1} \mathrm{H}$ NMR $\left(400 \mathrm{MHz} \mathrm{CDCl}_{3}\right) \delta: 10.74(\mathrm{bs}, 1 \mathrm{H}, \mathrm{NH}), 8.52(\mathrm{~d}, J=1.8 \mathrm{~Hz}, 1 \mathrm{H}$, H-4), $8.14(\mathrm{dd}, J=8.2,1.8 \mathrm{~Hz}, \mathrm{H}-6), 7.51\left(\mathrm{dd}, J=12.4,8.1 \mathrm{~Hz}, \mathrm{PTol}-\mathrm{H}_{\text {ortho }}\right), 7.46(\mathrm{~d}, J=8.1 \mathrm{~Hz}, 1 \mathrm{H}$, H-7), 7.41 (dd, $J=8.3,3.2 \mathrm{~Hz}$, PTol- $\left.\mathrm{H}_{\text {meta }}\right), 4.13-4.04(\mathrm{~m}, 1 \mathrm{H}, \mathrm{NCH}), 3.29-3.22(\mathrm{~m}, 1 \mathrm{H}, \mathrm{PCH}), 2.58(\mathrm{t}$, $\left.J=7.5 \mathrm{~Hz}, \mathrm{CH}_{2} \mathrm{CO}\right), 2.39\left(\mathrm{~s}, \mathrm{PTol}-\mathrm{CH}_{3}\right), 1.97-1.85\left(\mathrm{~m}, 1 \mathrm{H}, \mathrm{CH}_{2} \mathrm{CH}_{3}\right), 1.70-1.43(\mathrm{~m}, 7 \mathrm{H}), 1.32(\mathrm{t}, J=7.0$, $\left.\mathrm{CHCH}_{3}\right), 1.27-1.10(\mathrm{~m}, 10 \mathrm{H}), 0.74\left(\mathrm{t}, J=7.4 \mathrm{~Hz}, 3 \mathrm{H}, \mathrm{CH}_{2} \mathrm{CH}_{3}\right) .{ }^{13} \mathrm{C} \mathrm{NMR}\left(100 \mathrm{MHz}, \mathrm{CDCl}_{3}\right) \delta: 173.82$, 168.69, 168.51, 146.48 (d, $J=2.9 \mathrm{~Hz}, \mathrm{PAr}_{3}$ para), 145.42, 133.26 (d, $J=10.4 \mathrm{~Hz}, \mathrm{PAr}_{3}$ ortho), 132.85, 131.20 (d, $J=12.8 \mathrm{~Hz}, \mathrm{PAr}_{3}$ meta), 125.24, 124.28, 123.29, 114.84 (d, $J=88.7 \mathrm{~Hz}, \mathrm{PAr}_{3}$ ipso), 114.14, 48.64, 37.10, $30.28\left(\mathrm{~d}, J=15.5 \mathrm{~Hz}, \mathrm{CH}_{2} \mathrm{CH}_{2} \mathrm{CH}_{2} \mathrm{P}\right), 28.75,28.71,28.70,28.65,28.39,26.82,25.29,23.07(\mathrm{~d}, J=51.9 \mathrm{~Hz}$, $\left.\mathrm{CH}_{2} \mathrm{P}\right), 22.39\left(\mathrm{~d}, J=4.4 \mathrm{~Hz}, \mathrm{CH}_{2} \mathrm{CH}_{2} \mathrm{P}\right), 21.79\left(\mathrm{~d}, J=1.5 \mathrm{~Hz}, \mathrm{ArCH}_{3}\right), 18.37,11.21 .{ }^{31} \mathrm{P} \mathrm{NMR}(162 \mathrm{MHz}$, $\mathrm{CDCl}_{3}$ ) $\delta$ : 22.81. ES-HRMS $m / z$ for $\mathrm{C}_{44} \mathrm{H}_{54} \mathrm{~N}_{2} \mathrm{O}_{3} \mathrm{P}[\mathrm{M}]^{+}$: calcd. 689.3867 , found 689.3886 .

\subsubsection{General Procedure for the Synthesis of Phosphonium Phthalhydrazides}

A solution of the phosphonium phthalimide $(0.4 \mathrm{mmol})$ and hydrazine hydrate $(6 \mathrm{mmol})$ in ethanol $(15 \mathrm{~mL})$ was refluxed for $3 \mathrm{~h}$. After cooling, the volatiles were evaporated and the residue was subjected to column chromatography, yielding the corresponding phthalhydrazide.

(6-((1,4-Dioxo-1,2,3,4-tetrahydrophthalazin-5-yl)amino)-6-oxohexyl)triphenylphosphonium bromide (1a). From phthalimide 10a. Chromatography with methanol/DCM 5\% to 20\%. White solid (139 mg, 53\%). ${ }^{1} \mathrm{H}$ NMR $\left(200 \mathrm{MHz} \mathrm{CDCl}_{3}\right) \delta: 12.53(\mathrm{bs}, 1 \mathrm{H}, \mathrm{NH}), 12.06(\mathrm{bs}, 2 \mathrm{H}, \mathrm{NH}), 8.78(\mathrm{~d}, J=8.1 \mathrm{~Hz}, 1 \mathrm{H}$, H-6), 7.80-7.45 (m, 17H, Ar-H, H-7, H-8), 3.71-3.50 (m, 2H, $\left.\mathrm{CH}_{2} \mathrm{P}\right), 2.39-2.22$ (m, 2H, CH $\left.{ }_{2} \mathrm{CO}\right), 1.78-1.54$ $\left(\mathrm{m}, 6 \mathrm{H}, \mathrm{CH}_{2}\right) .{ }^{13} \mathrm{C}$ NMR $\left(50 \mathrm{MHz}, \mathrm{CDCl}_{3}\right) \delta: 172.02,159.90,154.47,140.90,135.13\left(\mathrm{~d}, J=1.8 \mathrm{~Hz}, \mathrm{PAr}_{3}\right.$ para), 134.21, 133.52 (d, $J=9.9 \mathrm{~Hz}, \mathrm{PAr}_{3}$ ortho), 130.54 (d, $J=12.5 \mathrm{~Hz}, \mathrm{PAr}_{3}$ meta), 127.91, 122.22, 119.59, 118.03 (d, $J=85.9 \mathrm{~Hz}$, ipso), 114.80, 37.95, $29.71\left(\mathrm{~d}, J=17.4 \mathrm{~Hz}, \mathrm{CH}_{2} \mathrm{CH}_{2} \mathrm{CH}_{2} \mathrm{P}\right), 24.40,22.25$ (d, $\left.J=49.6 \mathrm{~Hz}, \mathrm{CH}_{2} \mathrm{P}\right), 22.23\left(\mathrm{~d}, J=3.2 \mathrm{~Hz}, \mathrm{CH}_{2} \mathrm{CH}_{2} \mathrm{P}\right) .{ }^{31} \mathrm{P} \mathrm{NMR}\left(81 \mathrm{MHz}, \mathrm{CDCl}_{3}\right) \delta: 25.33$. ES-HRMS $\mathrm{m} / z$ for $\mathrm{C}_{32} \mathrm{H}_{31} \mathrm{~N}_{3} \mathrm{O}_{3} \mathrm{P}[\mathrm{M}]^{+}$: calcd. 536.2098, found 536.2097.

(6-((1,4-Dioxo-1,2,3,4-tetrahydrophthalazin-5-yl)amino)-6-oxohexyl)tri-p-tolylphosphonium bromide (1b). From phthalimide 10b. Chromatography with methanol/DCM 5\% to $20 \%$. White solid (84 mg, 32\%). ${ }^{1} \mathrm{H}$ NMR (200 MHz, $\left.\mathrm{CDCl}_{3}\right) \delta: 12.53$ (bs, $\left.1 \mathrm{H}, \mathrm{NH}\right), 12.66(\mathrm{bs}, 2 \mathrm{H}, \mathrm{NH}), 8.80(\mathrm{~d}, J=8.0 \mathrm{~Hz}, 1 \mathrm{H}$, $\mathrm{H}-6), 7.70(\mathrm{~d}, J=7.0 \mathrm{~Hz}, 1 \mathrm{H}, \mathrm{H}-8), 7.57-7.30(\mathrm{~m}, 13 \mathrm{H}, \mathrm{Ar}-\mathrm{H}, \mathrm{H}-7), 3.46-3.25\left(\mathrm{~m}, 2 \mathrm{H}, \mathrm{CH}_{2} \mathrm{P}\right), 2.41-2.18(\mathrm{~m}$, $\left.11 \mathrm{H}, \mathrm{ArCH}_{3}, \mathrm{CH}_{2} \mathrm{CO}\right), 1.78-1.52\left(\mathrm{~m}, 6 \mathrm{H}, \mathrm{CH}_{2}\right) .{ }^{13} \mathrm{C} \mathrm{NMR}\left(50 \mathrm{MHz}, \mathrm{CDCl}_{3}\right) \delta: 172.34,160.21,155.19$, 146.48 (d, $J=1.6 \mathrm{~Hz}, \mathrm{PAr}_{3}$ para), 140.93, 134.08, 133.44 (d, $J=10.4 \mathrm{~Hz}, \mathrm{PAr}_{3}$ ortho), 131.30 (d, $J=12.9 \mathrm{~Hz}$, $\mathrm{PAr}_{3}$ meta),128.51, 122.31, 119.94, 114.98 (d, $J=88.8 \mathrm{~Hz}, \mathrm{PAr}_{3}$ ipso), 115.25, 38.29, 29.93 (d, $J=16.0 \mathrm{~Hz}$, $\left.\mathrm{CH}_{2} \mathrm{CH}_{2} \mathrm{CH}_{2} \mathrm{P}\right), 24.58,22.74\left(\mathrm{~d}, J=49.2 \mathrm{~Hz}, \mathrm{CH}_{2} \mathrm{P}\right), 22.30\left(\mathrm{~d}, J=4.8 \mathrm{~Hz}, \mathrm{CH}_{2} \mathrm{CH}_{2} \mathrm{P}\right), 21.97 .{ }^{31} \mathrm{P} \mathrm{NMR}$ $\left(81 \mathrm{MHz}, \mathrm{CDCl}_{3}\right) \delta: 25.23$. ES-HRMS $m / z$ for $\mathrm{C}_{35} \mathrm{H}_{37} \mathrm{~N}_{3} \mathrm{O}_{3} \mathrm{P}[\mathrm{M}]^{+}$: calcd. 578.2657, found 578.2619.

(11-((1,4-Dioxo-1,2,3,4-tetrahydrophthalazin-5-yl)amino)-11-oxoundecyl)triphenylphosphonium bromide (1c). From phthalimide 10c. Chromatography with methanol/DCM 5\% to 20\%. White solid $(135 \mathrm{mg}$, 49\%). ${ }^{1} \mathrm{H}$ NMR (200 MHz, DMSO- $\left.d_{6}\right) \delta: 15.58$ (bs, $\left.1 \mathrm{H}, \mathrm{NH}\right), 8.74(\mathrm{~d}, J=7.7 \mathrm{~Hz}, 1 \mathrm{H}, \mathrm{H}-6), 7.90-7.70$ (m, $15 \mathrm{H}, \mathrm{ArH}), 7.62(\mathrm{~d}, J=7.7 \mathrm{~Hz}, 1 \mathrm{H}, \mathrm{H}-8), 7.49(\mathrm{t}, J=7.7 \mathrm{~Hz}, 1 \mathrm{H}, \mathrm{H}-7), 3.60-3.45\left(\mathrm{~m}, 2 \mathrm{H}, \mathrm{CH}_{2} \mathrm{P}\right), 2.28(\mathrm{t}$, $\left.J=7.1 \mathrm{~Hz}, 2 \mathrm{H}, \mathrm{CH}_{2} \mathrm{CO}\right), 1.65-1.10\left(\mathrm{~m}, 16 \mathrm{H}, \mathrm{CH}_{2}\right) .{ }^{13} \mathrm{C}$ NMR $\left(50 \mathrm{MHz}, \mathrm{DMSO}-d_{6}\right) \delta: 171.09,160.37$, 156.81, 140.29, 134.84 (d, J = 2.6 Hz, PAr 3 para), 133.58 (d, J = $10.1 \mathrm{~Hz}, \mathrm{PAr}_{3}$ ortho), 130.34, 130.22 (d, $J=12.4 \mathrm{~Hz}, \mathrm{PAr}_{3}$ meta), 129.99, 119.50, 118.62 (d, $J=85.6 \mathrm{~Hz}, \mathrm{PAr}_{3}$ ipso), 118.59, 117.36, 38.10, 30.72, $29.67\left(\mathrm{~d}, \mathrm{~J}=16.5 \mathrm{~Hz}, \mathrm{CH}_{2} \mathrm{CH}_{2} \mathrm{CH}_{2} \mathrm{P}\right), 28.59(2 \mathrm{C}), 28.51,27.95,25.03,21.67\left(\mathrm{~d}, J=4.2 \mathrm{~Hz}, \mathrm{CH}_{2} \mathrm{CH}_{2} \mathrm{P}\right)$, 
$20.09\left(\mathrm{~d}, J=49.4 \mathrm{~Hz}, \mathrm{CH}_{2} \mathrm{P}\right) .{ }^{31} \mathrm{P}$ NMR $\left(162 \mathrm{MHz}, \mathrm{CDCl}_{3}\right) \delta: 23.39$. ES-HRMS $m / z$ for $\mathrm{C}_{37} \mathrm{H}_{41} \mathrm{~N}_{3} \mathrm{O}_{3} \mathrm{P}$ $[\mathrm{M}]^{+}$: calcd. 606.2880 , found 606.2888 .

(11-((1,4-Dioxo-1,2,3,4-tetrahydrophthalazin-5-yl)amino)-11-oxoundecyl)tri-p-tolylphosphonium bromide (1d). From phthalimide 10d. Chromatography with methanol/DCM 5\% to 20\%. White solid $(108 \mathrm{mg}$, 37\%). ${ }^{1} \mathrm{H}$ NMR $\left(200 \mathrm{MHz}, \mathrm{CDCl}_{3}\right) \delta: 12.74(\mathrm{bs}, 1 \mathrm{H}, \mathrm{NH}), 8.95(\mathrm{~d}, J=8.2 \mathrm{~Hz}, 1 \mathrm{H}, \mathrm{H}-6), 7.77$ (d, J = $7.7 \mathrm{~Hz}$, $1 \mathrm{H}, \mathrm{H}-8), 7.62(\mathrm{t}, J=8.1 \mathrm{~Hz}, 1 \mathrm{H}, \mathrm{H}-7), 7.55-7.36(\mathrm{~m}, 15 \mathrm{H}, \mathrm{ArH}), 3.36-3.22\left(\mathrm{~m}, 2 \mathrm{H}, \mathrm{CH}_{2} \mathrm{P}\right), 2.42-2.27$ $\left(\mathrm{m}, 11 \mathrm{H}, \mathrm{ArCH}_{3}, \mathrm{CH}_{2} \mathrm{CO}\right), 1.72-1.04\left(\mathrm{~m}, 16 \mathrm{H}, \mathrm{CH}_{2}\right) .{ }^{13} \mathrm{C} \mathrm{NMR}\left(50 \mathrm{MHz}, \mathrm{CDCl}_{3}\right) \delta: 172.80,159.66$, 154.97, 146.34 (d, $J=3.0 \mathrm{~Hz}, \mathrm{PAr}_{3}$ para), 141.21, 134.06, 133.24 (d, $J=10.3 \mathrm{~Hz}, \mathrm{PAr}_{3}$ ortho), 131.15 (d, $J=12.9 \mathrm{~Hz}, \mathrm{PAr}_{3}$ meta), 128.28, 122.41, 119.66, 115.12, 114.86 (d, $J=88.7 \mathrm{~Hz}, \mathrm{PAr}_{3}$ ipso), 38.62, 30.38 (d, $\left.J=15.7 \mathrm{~Hz}, \mathrm{CH}_{2} \mathrm{CH}_{2} \mathrm{CH}_{2} \mathrm{P}\right), 28.91(2 \mathrm{C}), 28.87,28.83,28.77,25.31,22.82\left(\mathrm{~d}, J=51.6 \mathrm{~Hz}, \mathrm{CH}_{2} \mathrm{P}\right), 22.49(\mathrm{~d}$, $\left.J=4.2 \mathrm{~Hz}, \mathrm{CH}_{2} \mathrm{CH}_{2} \mathrm{P}\right), 21.81\left(\mathrm{~d}, J=1.2 \mathrm{~Hz}, \mathrm{ArCH}_{3}\right) .{ }^{31} \mathrm{P} \mathrm{NMR}\left(81 \mathrm{MHz}, \mathrm{CDCl}_{3}\right) \delta: 23.82$. ES-HRMS $m / z$ for $\mathrm{C}_{40} \mathrm{H}_{47} \mathrm{~N}_{3} \mathrm{O}_{3} \mathrm{P}[\mathrm{M}]^{+}$: calcd. 648.3350, found 648.3437 .

Tricyclohexyl(11-((1,4-dioxo-1,2,3,4-tetrahydrophthalazin-5-yl)amino)-11-oxoundecyl)phosphonium bromide (1e). From phthalimide 10e. Chromatography with methanol/DCM 5\% to 20\%. White solid (56 mg, 20\%). ${ }^{1} \mathrm{H}$ NMR (400 MHz, $\left.\mathrm{CDCl}_{3}\right) \delta: 12.67$ (bs, $\left.1 \mathrm{H}, \mathrm{NH}\right), 8.98(\mathrm{~d}, J=8.3 \mathrm{~Hz}, 1 \mathrm{H}, \mathrm{H}-6), 7.81(\mathrm{~d}$, $J=7.8 \mathrm{~Hz}, 1 \mathrm{H}, \mathrm{H}-8), 7.69(\mathrm{t}, J=8.1 \mathrm{~Hz}, 1 \mathrm{H}, \mathrm{H}-7), 2.53(\mathrm{q}, J=11.5 \mathrm{~Hz}, 3 \mathrm{H}, \mathrm{PCyCH}), 2.40(\mathrm{t}, J=7.5 \mathrm{~Hz}$, $\left.2 \mathrm{H}, \mathrm{COCH}_{2}\right), 2.38-2.30\left(\mathrm{~m}, 2 \mathrm{H}, \mathrm{PCH}_{2}\right), 2.01-1.19\left(\mathrm{~m}, 46 \mathrm{H}, \mathrm{CH}_{2}\right) .{ }^{13} \mathrm{C} \mathrm{NMR}\left(100 \mathrm{MHz}, \mathrm{CDCl}_{3}\right) \delta: 172.81$, $159.82,154.77,141.40,134.35,128.09,122.63,119.67,115.09,38.73,31.18$ (d, $J=13.6 \mathrm{~Hz}, \mathrm{CH}_{2} \mathrm{CH}_{2} \mathrm{CH}_{2} \mathrm{P}$ ), $30.02\left(\mathrm{~d}, J=40.3 \mathrm{~Hz}, \mathrm{PCy}_{3}-\mathrm{C} 1\right), 29.15,28.98,28.94,28.86,28.82,27.25\left(\mathrm{~d}, J=3.9 \mathrm{~Hz}, \mathrm{PCy}_{3}-\mathrm{C} 2\right), 26.53$ $\left(\mathrm{d}, J=11.7 \mathrm{~Hz}, \mathrm{PCy}_{3}-\mathrm{C} 3\right), 25.47,25.35,22.79\left(\mathrm{~d}, J=5.2 \mathrm{~Hz}, \mathrm{CH}_{2} \mathrm{CH}_{2} \mathrm{P}\right), 15.82\left(\mathrm{~d}, J=42.5 \mathrm{~Hz}, \mathrm{CH}_{2} \mathrm{P}\right)$. ${ }^{31} \mathrm{P}$ NMR $\left(81 \mathrm{MHz}, \mathrm{CDCl}_{3}\right) \delta$ : 32.71. ES-HRMS $\mathrm{m} / z$ for $\mathrm{C}_{37} \mathrm{H}_{59} \mathrm{~N}_{3} \mathrm{O}_{3} \mathrm{P}[\mathrm{M}]^{+}$: calcd. 624.4289 , found 624.4295 .

(6-((1,4-dioxo-1,2,3,4-tetrahydrophthalazin-6-yl)amino)-6-oxohexyl)tri-p-tolylphosphonium bromide (12a). From phthalimide 11a. Chromatography with methanol/DCM $5 \%$ to $20 \%$. White solid $(190 \mathrm{mg}$, 72\%). ${ }^{1} \mathrm{H}$ NMR (200 MHz, DMSO- $d_{6}$ ) $\delta: 11.46$ (bs, 2H, NHNH), 10.63 (bs, 1H, NHCO), 8.42 (s, 1H, H-5), 8.03-7.94 (m, 2H, H-7, H-8), 7.68-7.55 (m, 12H, ArH), 3.56-3.45 (m, 2H, $\left.\mathrm{CH}_{2} \mathrm{P}\right), 2.46-2.30(\mathrm{~m}, 11 \mathrm{H}$, $\left.\mathrm{ArCH}_{3}, \mathrm{CH}_{2} \mathrm{CO}\right), 1.60-1.45\left(\mathrm{~m}, 6 \mathrm{H}, \mathrm{CH}_{2}\right) .{ }^{13} \mathrm{C}$ NMR (50 MHz, DMSO- $\left.d_{6}\right) \delta: 172.13,155.00,154.50$, $145.53\left(\mathrm{~d}, J=2.8 \mathrm{~Hz}, \mathrm{PAr}_{3}\right.$ para), 143.10, 133.46 (d, $J=10.4 \mathrm{~Hz}, \mathrm{PAr}_{3}$ ortho), 130.80 (d, $J=12.8 \mathrm{~Hz}$, $\mathrm{PAr}_{3}$ meta),128.18, 126.24, 123.29, 122.33, 115.51 (d, $J=88.2 \mathrm{~Hz}, \mathrm{PAr}_{3}$ ipso), 113.28, 36.08, 29.51 (d, $\left.J=17.5 \mathrm{~Hz}, \mathrm{CH}_{2} \mathrm{CH}_{2} \mathrm{CH}_{2} \mathrm{P}\right), 24.30,21.67\left(\mathrm{~d}, J=5.0 \mathrm{~Hz}, \mathrm{CH}_{2} \mathrm{CH}_{2} \mathrm{P}\right), 21.28\left(\mathrm{~d}, J=1.4 \mathrm{~Hz}, \mathrm{ArCH}_{3}\right), 20.52$ $\left(\mathrm{d}, J=52.0 \mathrm{~Hz}, \mathrm{CH}_{2} \mathrm{P}\right) .{ }^{31} \mathrm{P}$ NMR $\left(81 \mathrm{MHz}, \mathrm{DMSO}-d_{6}\right) \delta: 24.04$. ES-HRMS $m / z$ for $\mathrm{C}_{35} \mathrm{H}_{37} \mathrm{~N}_{3} \mathrm{O}_{3} \mathrm{P}[\mathrm{M}]^{+}$: calcd. 578.2567, found 578.2627.

Tricyclohexyl(6-((1,4-dioxo-1,2,3,4-tetrahydrophthalazin-6-yl)amino)-6-oxohexyl)phosphonium bromide (12b). From phthalimide 11b. Chromatography with methanol/DCM 5\% to 20\%. White solid $(157 \mathrm{mg}$, 62\%). ${ }^{1} \mathrm{H}$ NMR (200 MHz, DMSO- $\left.d_{6}\right) \delta: 11.25$ (bs, $\left.1 \mathrm{H}, \mathrm{NHCO}\right), 8.56(\mathrm{~s}, 1 \mathrm{H}, \mathrm{H}-5), 8.11$ (d, J = $8.7 \mathrm{~Hz}$, $1 \mathrm{H}, \mathrm{H}-7), 7.97(\mathrm{~d}, J=8.6 \mathrm{~Hz}, 1 \mathrm{H}, \mathrm{H}-8), 2.59-2.43(\mathrm{~m}, 5 \mathrm{H}, \mathrm{PCH}), 2.32-2.15\left(\mathrm{~m}, 2 \mathrm{H}, \mathrm{COCH}_{2}\right), 2.03-1.26$ $\left(\mathrm{m}, 36 \mathrm{H}, \mathrm{CH}_{2}\right) .{ }^{13} \mathrm{C}$ NMR $\left(50 \mathrm{MHz}, \mathrm{DMSO}-d_{6}\right) \delta: 172.31,155.00,154.39,143.20,128.18,126.15,123.34$, 122.35, 113.32, 36.03, 30.11 (d, $J=13.3 \mathrm{~Hz}, \mathrm{CH}_{2} \mathrm{CH}_{2} \mathrm{CH}_{2} \mathrm{P}$ ), 28.54 (d, J = 41.2 Hz, $\mathrm{CH}_{2} \mathrm{P}$-cyclo), 26.11 (d, $J=4.2 \mathrm{~Hz}, \mathrm{CH}_{2} \mathrm{CH}_{2} \mathrm{P}$-cyclo), 25.94 (d, $J=12.8 \mathrm{~Hz}, \mathrm{CH}_{2} \mathrm{CH}_{2} \mathrm{CH}_{2} \mathrm{P}$-cyclo), 25.02, 24.26, 21.44 (d, $\left.J=2.8 \mathrm{~Hz}, \mathrm{CH}_{2} \mathrm{CH}_{2} \mathrm{P}\right), 14.28\left(\mathrm{~d}, J=44.0 \mathrm{~Hz}, \mathrm{CH}_{2} \mathrm{P}\right) .{ }^{31} \mathrm{P}$ NMR $\left(81 \mathrm{MHz}\right.$, DMSO- $\left.d_{6}\right) \delta:$ 32.47. ES-HRMS $\mathrm{m} / \mathrm{z}$ for $\mathrm{C}_{32} \mathrm{H}_{49} \mathrm{~N}_{3} \mathrm{O}_{3} \mathrm{P}[\mathrm{M}]^{+}$: calcd. 554.3506, found 554.3556 .

(11-((1,4-Dioxo-1,2,3,4-tetrahydrophthalazin-6-yl)amino)-11-oxoundecyl)tri-p-tolylphosphonium bromide (12c). From phthalimide 11c. Chromatography with methanol/DCM 5\% to 20\%. White solid (108 mg, 37\%). ${ }^{1} \mathrm{H}$ NMR (200 MHz, DMSO- $\left.d_{6}\right) \delta: 11.44$ (bs, 2H, NHNH), 10.47 (bs, $\left.1 \mathrm{H}, \mathrm{NHCO}\right), 8.40(\mathrm{~s}, 1 \mathrm{H}$, $\mathrm{H}-5), 8.03-7.94(\mathrm{~m}, 2 \mathrm{H}, \mathrm{H}-7, \mathrm{H}-8), 7.68-7.53(\mathrm{~m}, 12 \mathrm{H}, \mathrm{ArH}), 3.52-3.43\left(\mathrm{~m}, 2 \mathrm{H}, \mathrm{CH}_{2} \mathrm{P}\right), 2.44-2.33(\mathrm{~m}, 11 \mathrm{H}$, $\left.\mathrm{ArCH}_{3}, \mathrm{CH}_{2} \mathrm{CO}\right), 1.65-1.16\left(\mathrm{~m}, 16 \mathrm{H}, \mathrm{CH}_{2}\right) .{ }^{13} \mathrm{C}$ NMR (50 MHz, DMSO-d 6 ) $\delta: 172.32,154.93,154.38$, 145.55 (d, $J=3.0 \mathrm{~Hz}, \mathrm{PAr}_{3}$ para), 143.17, 133.44 (d, J=10.4 Hz, PAr 3 ortho), 130.80 (d, J=12.8 Hz, $\mathrm{PAr}_{3}$ meta), 128.15, 126.24, 123.31, 122.23, 115.54 (d, $J=88.2 \mathrm{~Hz}, \mathrm{PAr}_{3}$ ipso), 113.26, 36.45, 29.79 (d, 
$\left.J=16.6 \mathrm{~Hz}, \mathrm{CH}_{2} \mathrm{CH}_{2} \mathrm{CH}_{2} \mathrm{P}\right), 28.75,28.68,28.63,28.13,25.02,21.76\left(\mathrm{~d}, J=3.5 \mathrm{~Hz}, \mathrm{CH}_{2} \mathrm{CH}_{2} \mathrm{P}\right), 21.28(\mathrm{~d}$, $\left.J=1.5 \mathrm{~Hz}, \mathrm{ArCH}_{3}\right), 20.46\left(\mathrm{~d}, J=50.4 \mathrm{~Hz}, \mathrm{CH}_{2} \mathrm{P}\right) .{ }^{31} \mathrm{P}$ NMR $\left(81 \mathrm{MHz}, \mathrm{DMSO}-d_{6}\right) \delta: 24.13$. ES-HRMS $m / z$ for $\mathrm{C}_{40} \mathrm{H}_{47} \mathrm{~N}_{3} \mathrm{O}_{3} \mathrm{P}[\mathrm{M}]^{+}$: calcd. 648.3350, found 648.3401 .

\subsubsection{Synthesis of 3-Heptanamidophthalic Acid (14)}

Synthesis of 4-aminoisobenzofuran-1,3-dione 15 [50]. A stirred solution of 3-nitrophthalic anhydride 4a ( $4.5 \mathrm{~g}, 23 \mathrm{mmol})$ in THF (40 mL) was degassed (Ar) for $30 \mathrm{~min} .10 \% \mathrm{Pd} / \mathrm{C}(200 \mathrm{mg})$ was added, then bubbled with $\mathrm{H}_{2}$ for a while and the mixture was stirred under $\mathrm{H}_{2}$ atmosphere (20 bar) for $18 \mathrm{~h}$. The mixture was filtered through celite, washed with ethyl acetate and the filtrate was concentrated. The solid residue was washed with dichloromethane (extracting $\approx 1 \mathrm{~g}$ of a mixture containing the product), silica gel was added in the combined washings, the solvent was evaporated and the residue was dry-loaded onto column chromatography (dichloromethane), affording 3-aminophthalic anhydride 15 as yellow solid (488 mg, 13\%). ${ }^{1} \mathrm{H}$ NMR $\left(200 \mathrm{MHz}, \mathrm{DMSO}-d_{6}\right) \delta: 7.57(\mathrm{t}, J=7.8 \mathrm{~Hz}, 1 \mathrm{H}, \mathrm{H}-6), 7.11(\mathrm{~d}$, $J=7.0 \mathrm{~Hz}, 1 \mathrm{H}, \mathrm{H}-5), 7.09$ (d, $J=8.5 \mathrm{~Hz}, 1 \mathrm{H}, \mathrm{H}-4), 6.83\left(\mathrm{~s}, 2 \mathrm{H}, \mathrm{NH}_{2}\right) .{ }^{13} \mathrm{C}$ NMR $\left(50 \mathrm{MHz}, \mathrm{DMSO}-d_{6}\right)$ $\delta: 164.00,163.92,148.33,137.21,131.47,122.25,112.68,108.01$. ES-MS $m / z$ for $\mathrm{C}_{8} \mathrm{H}_{5} \mathrm{NO}_{3}[\mathrm{M}]^{+}$: calcd. 163.0, found 163.0. A solution of heptanoic acid ( $263 \mathrm{mg}, 2.024 \mathrm{mmol})$ in oxalyl chloride (1 mL) was stirred for $5 \mathrm{~h}$ under Ar. Then, the volatiles were evaporated to dryness under reduced pressure at room temperature. The residue was dissolved in dry dichloromethane $(1 \mathrm{~mL})$ and added dropwise to a cooled $\left(0{ }^{\circ} \mathrm{C}\right)$ solution of the anhydride $15(300 \mathrm{mg}, 1.84 \mathrm{mmol})$ and pyridine $(474 \mu \mathrm{L}, 5.52 \mathrm{mmol})$ in dichloromethane $(5 \mathrm{~mL})$ under Ar. The resulting mixture was stirred at r.t. for $48 \mathrm{~h}$. Water $(40 \mathrm{~mL})$ was added and the aqueous phase was washed with dichloromethane $(2 \times 20 \mathrm{~mL})$ and ethyl acetate $(3 \times 20 \mathrm{~mL})$. The combined ethyl acetate washings were dried $\left(\mathrm{Na}_{2} \mathrm{SO}_{4}\right)$, solvent was evaporated and the residue was subjected to column chromatography ( $0 \%$ to $20 \%$ methanol/DCM) yielding phthalic acid 14 as off-white solid (17 mg, 3\%). ${ }^{1} \mathrm{H}$ NMR (200 MHz, DMSO- $\left.d_{6}\right) \delta: 11.92(\mathrm{bs}, 1 \mathrm{H}, \mathrm{NH}), 8.40(\mathrm{~d}$, $J=8.2, \mathrm{~Hz}, 1 \mathrm{H}, \mathrm{H}-4), 7.38-7.27(\mathrm{~m}, 2 \mathrm{H}, \mathrm{H}-5, \mathrm{H}-6), 2.28\left(\mathrm{t}, J=7.3 \mathrm{~Hz}, 2 \mathrm{H}, \mathrm{COCH}_{2}\right), 1.64-1.52(\mathrm{~m}, 2 \mathrm{H}$, $\left.\mathrm{COCH}_{2} \mathrm{CH}_{2}\right), 1.33-1.24(\mathrm{~m}, 6 \mathrm{H}), 0.86\left(\mathrm{t}, J=6.3 \mathrm{~Hz}, 3 \mathrm{H}\right.$, hexyl- $\left.\mathrm{CH}_{3}\right) .{ }^{13} \mathrm{C}$ NMR $\left(50 \mathrm{MHz}, \mathrm{DMSO}-d_{6}\right) \delta$ : 173.29, 173.20, 172.82, 155.51, 135.85, 128.47, 127.95, 123.88, 123.34, 37.27, 31.31, 28.58, 25.34, 22.18, 12.98 . ES-HRMS $m / z$ for $\mathrm{C}_{15} \mathrm{H}_{18} \mathrm{NO}_{5}[\mathrm{M}-\mathrm{H}]^{-}$: calcd. 292.1185, found 292.1188.

\section{Conclusions}

A series of phosphonium-functionalized amino-acylated luminol and isoluminol derivatives was synthesized. Direct acylation of (iso)luminol resulted in inseparable mixtures, so their preparation was accomplished through the acylation of the corresponding, easily-accessible phthalimides, followed by hydrazinolysis. In this way, the targeted derivatives were isolated in a scalable and repeatable manner. All compounds were fully characterized using NMR and HRMS spectroscopies. The $\mathrm{H}_{2} \mathrm{O}_{2}$-triggered chemiluminescence of the synthesized compounds was investigated under alkaline conditions and compared to that of the parent compound. In general, all amino-acylated luminol derivatives exhibit a CL quantum yield markedly lower to that of luminol. The remarkable decrease in the CL quantum yield is attributed to both the weaker fluorescence of the corresponding phthalates and, more importantly, to the poorer electron-donating nature of the aromatic ring substituent, which results in a lower yield of the CL-triggering oxidation reaction. Thus, while the task of attaching a phosphonium cation on luminol is feasible, the amino-acylation approach, towards (iso)luminol functionalization, affords inefficient chemiluminescent derivatives. As a result, different functionalization routes have to be considered.

Supplementary Materials: NMR spectra for the prepared compounds are available online at http://www.mdpi. com/1420-3049/24/21/3957/s1.

Author Contributions: Conceptualization, G.C.V., G.R., M.A.M., and M.C.C.; Methodology, G.C.V., G.R., M.A.M., and M.C.C.; Validation, G.C.V. and G.R.; Investigation, A.P., I.D., and M.C.C.; Resources, G.C.V. and M.A.M.; Writing-original draft preparation, G.R.; Writing—review and editing, G.C.V. and M.A.M.; Visualization, G.R.; Supervision, G.C.V., G.R., and M.A.M.; Project administration, G.C.V. and M.A.M.; Funding acquisition, G.C.V., A.P., and M.A.M. 
Funding: This project was financially supported by the European Union's Horizon 2020 framework program for research and innovation under grant agreement no. 712921, as well as a Greek State Scholarships Foundation (I.K.Y.) fellowship to A.P.

Conflicts of Interest: The authors declare no conflict of interest.

\section{References and Notes}

1. Gundermann, K.D. Chemiluminescence in organic compounds. Angew. Chem., Int. Ed. Engl. 1965, 4, 566-573. [CrossRef]

2. Rongen, H.A.H.; Hoetelmans, R.M.W.; Bult, A.; Van Bennekom, W.P. Chemiluminescence and immunoassays. J. Pharm. Biomed. Anal. 1994, 12, 433-462. [CrossRef]

3. Dodeigne, C.; Thunus, L.; Lejeune, R. Chemiluminescence as diagnostic tool. A review. Talanta 2000, 51, 415-439. [CrossRef]

4. Barnett, N.W.; Francis, P.S. Chemiluminescence: Liquid-Phase. In Encyclopedia of Analytical Science, 2nd ed.; Worsfold, P., Townshend, A., Poole, C., Eds.; Oxford University Press: New York, NY, USA, 2005; pp. 511-521.

5. Murphy, M.P.; Holmgren, A.; Larsson, N.-G.; Halliwell, B.; Chang, C.J.; Kalyanaraman, B.; Rhee, S.G.; Thornalley, P.J.; Partridge, L.; Gems, D.; et al. Unraveling the biological roles of reactive oxygen species. Cell Metab. 2011, 13, 361-366. [CrossRef] [PubMed]

6. Schieber, M.; Chandel, N.S. ROS function in redox signaling and oxidative stress. Curr. Biol. 2014, 24, 453-462. [CrossRef] [PubMed]

7. Zhang, Y.; Dai, M.; Yuan, Z. Methods for the detection of reactive oxygen species. Anal. Methods 2018, 10, 4625-4638. [CrossRef]

8. Blázquez-Castro, A.; Breitenbach, T.; Ogilby, P.R. Singlet oxygen and ROS in a new light: Low-dose subcellular photodynamic treatment enhances proliferation at the single cell level. Photochem. Photobiol. Sci. 2014, 13, 1235-1240. [CrossRef]

9. Gomes, A.; Fernandes, E.; Lima, J.L.F.C. Fluorescence probes used for detection of reactive oxygen species. J. Biochem. Bioph. Meth. 2005, 65, 45-80. [CrossRef]

10. Chen, X.; Tian, X.; Shin, I.; Yoon, J. Fluorescent and luminescent probes for detection of reactive oxygen and nitrogen species. Chem. Soc. Rev. 2011, 40, 4783-4804. [CrossRef]

11. Brand, M.D. Mitochondrial generation of superoxide and hydrogen peroxide as the source of mitochondrial redox signaling. Free Radical Biol. Med. 2016, 100, 14-31. [CrossRef]

12. Cheng, G.; Zielonka, M.; Dranka, B.; Kumar, S.N.; Myers, C.R.; Bennett, B.; Garces, A.M.; Dias Duarte Machado Luiz, G.; Thiebaut, D.; Ouari, O.; et al. Detection of mitochondria-generated reactive oxygen species in cells using multiple probes and methods: Potentials, pitfalls, and the future. J. Biol. Chem. 2018, 293, 10363-10380. [CrossRef] [PubMed]

13. Murphy, M.P. How mitochondria produce reactive oxygen species. Biochem. J. 2009, 417, 1-13. [CrossRef] [PubMed]

14. Su, Y.; Song, H.; Lv, Y. Recent advances in chemiluminescence for reactive oxygen species sensing and imaging analysis. Microchem. J. 2019, 146, 83-97. [CrossRef]

15. Hananya, N.; Green, O.; Blau, R.; Satchi-Fainaro, R.; Shabat, D. A Highly efficient chemiluminescence probe for the detection of singlet oxygen in living cells. Angew. Chem. Int. Ed. 2017, 56, 11793-11796. [CrossRef]

16. Prolo, C.; Rios, N.; Piacenza, L.; Álvarez, M.N.; Radi, R. Fluorescence and chemiluminescence approaches for peroxynitrite detection. Free Radic. Biol. Med. 2018, 128, 59-68. [CrossRef]

17. Vladimirov, Y.A.; Proskurnina, E.V. Free radicals and cell chemiluminescence. Biochem. (Mosc.) 2009, 74, 1545-1566. [CrossRef]

18. Yamaguchi, S.; Kishikawa, N.; Ohyama, K.; Ohba, Y.; Kohno, M.; Masuda, T.; Takadate, A.; Nakashima, N.; Kuroda, N. Evaluation of chemiluminescence reagents for selective detection of reactive oxygen species. Anal. Chim. Acta 2010, 665, 74-78. [CrossRef]

19. Barni, F.; Lewis, S.W.; Berti, A.; Miskelly, G.M.; Lago, G. Forensic application of the luminol reaction as a presumptive test for latent blood detection. Talanta 2007, 72, 896-913. [CrossRef]

20. Marquette, C.A.; Blum, L.J. Applications of the luminol chemiluminescent reaction in analytical chemistry. Anal. Bioanal. Chem. 2006, 385, 546-554. [CrossRef] 
21. Khan, P.; Idrees, D.; Moxley, M.A.; Corbett, J.A.; Ahmad, F.; von Figura, G.; Sly, W.S.; Waheed, A.; Hassan, M.I. Luminol-Based Chemiluminescent Signals: Clinical and Non-clinical Application and Future Uses. Appl. Biochem. Biotechnol. 2014, 173, 333-355. [CrossRef]

22. Schmitz, A.J. Ueber das Hydrazid der Trimesinsäure und der Hemimellithsäure. Ph.D. Thesis, Heidelberg University, Heidelberg, Germany, 1902.

23. Albrecht, H.O. Über die chemiluminescenz des aminophthalsäurehydrazids. Z. Physik. Chem. 1928, 136, 321-330. [CrossRef]

24. Huntress, E.; Stanley, L.; Parker, A. The preparation of 3-aminophthalhydrazide for use in the demonstration of chemiluminescence. J. Am. Chem. Soc. 1934, 56, 241-242. [CrossRef]

25. Specht, W. Die Chemiluminescenz des hämins, ein hilfsmittel zur auffindung und erkennung forensisch wichtiger blutspuren. Angew. Chem. 1937, 50, 155-157. [CrossRef]

26. Zhang, N.; Francis, K.P.; Prakash, A.; Ansaldi, D. Enhanced detection of myeloperoxidase activity in deep tissues through luminescent excitation of near-infrared nanoparticles. Nat. Med. 2013, 19, 500. [CrossRef]

27. Gross, S.; Gammon, S.T.; Moss, B.L.; Rauch, D.; Harding, J.; Heinecke, J.W.; Ratner, L.; Piwnica-Worms, D. Bioluminescence imaging of myeloperoxidase activity in vivo. Nat. Med. 2009, 15, 455. [CrossRef]

28. Guo, J.; Tao, H.; Dou, Y.; Li, L.; Xu, X.; Zhang, Q.; Cheng, J.; Han, S.; Huang, J.; Li, X.; et al. Myeloperoxidase-responsive and biodegradable luminescent material for real-time imaging of inflammatory diseases. Mater. Today 2017, 20, 493-500. [CrossRef]

29. Zielonka, J.; Joseph, J.; Sikora, A.; Hardy, M.; Ouari, O.; Vasquez-Vivar, J.; Cheng, G.; Lopez, M.; Kalyanaraman, B. Mitochondria-Targeted triphenylphosphonium-based compounds: Syntheses, mechanisms of action, and therapeutic and diagnostic applications. Chem. Rev. 2017, 117, 10043-10120. [CrossRef]

30. Ong, H.C.; Hu, Z.; Coimbra, J.T.S.; Ramos, M.J.; Kon, O.L.; Xing, B.; Yeow, E.K.L.; Fernandes, P.A.; García, F. Enabling mitochondrial uptake of lipophilic dications using methylated triphenylphosphonium moieties. Inorg. Chem. 2019, 58, 8293-8299. [CrossRef]

31. Murphy, M.P.; Smith, R.A.J. Targeting antioxidants to mitochondria by conjugation to lipophilic cations. Annu. Rev. Pharm. 2007, 47, 629-656. [CrossRef]

32. Omote, Y.; Miyake, T.; Ohmori, S.; Sugiyama, N. The Chemiluminescence of Acyl Luminols. Bull. Chem. Soc. Jpn. 1966, 39, 932-935. [CrossRef]

33. Yoshimori, O.; Takeo, M.; Seiya, O.; Noboru, S. The chemiluminescence of luminol and acetyl luminol. Bull. Chem. Soc. Jpn. 1967, 40, 899-903.

34. Gundermann, K.-D.; Drawert, M. Konstitution und chemilumineszenz, i. sterische resonanzhinderung bei alkylierten amino-phthalhydraziden. Chem. Ber. 1962, 95, 2018-2026. [CrossRef]

35. Liu, J.-L.; Zhao, M.; Zhuo, Y.; Chai, Y.-Q.; Yuan, R. Highly efficient intramolecular electrochemiluminescence energy transfer for ultrasensitive bioanalysis of aflatoxin M1. Chem. Eur. J. 2017, 23, 1853-1859. [CrossRef] [PubMed]

36. Zhang, W.-Z.; Du, Z.-B.; Song, B.; Ye, Z.-Q.; Yuan, J.-L. Development of a triple channel detection probe for hydrogen peroxide. Chinese Chem. Lett. 2015, 26, 1465-1469. [CrossRef]

37. Shibata, T.; Yoshimura, H.; Yamayoshi, A.; Tsuda, N.; Dragusha, S. Hydrazide derivatives of luminol for chemiluminescence-labelling of macromolecules. Chem. Pharm. Bull. 2019, 67, 772-774. [CrossRef]

38. Theodossiou, T.A.; Sideratou, Z.; Tsiourvas, D.; Paleos, C.M. A novel mitotropic oligolysine nanocarrier: Targeted delivery of covalently bound D-Luciferin to cell mitochondria. Mitochondrion 2011, 11, 982-986. [CrossRef]

39. Selinger, Z.; Lapidot, Y. Synthesis of fatty acid anhydrides by reaction with dicyclohexylcarbodiimide. J. Lipid Res. 1966, 7, 174-175.

40. Chu, W.; Tu, Z.; McElveen, E.; Xu, J.; Taylor, M.; Luedtke, R.R.; Mach, R.H. Synthesis and in vitro binding of $\mathrm{N}$-phenyl piperazine analogs as potential dopamine D3 receptor ligands. Bioorg. Med. Chem. 2005, 13, 77-87. [CrossRef]

41. Karatani, H. Microenvironmental effects of water-soluble polymers on the chemiluminescence of luminol and its analogs. Bull. Chem. Soc. Jpn. 1987, 60, 2023-2029. [CrossRef]

42. Schroeder, H.R.; Boguslaski, R.C.; Carrico, R.J.; Buckler, R.T. Monitoring specific protein-binding reactions with chemiluminescence. in methods in enzymology. Acad. Press 1978, 57, 424-445.

43. Neelakantan, S.; Surjawan, I.; Karacelik, H.; Hicks, C.L.; Crooks, P.A. Synthesis of novel isoluminol probes and their use in rapid bacterial assays. Bioorg. Med. Chem. Lett. 2009, 19, 5722-5726. [CrossRef] [PubMed] 
44. Yang, L.; Liu, X.; Gao, L.; Qi, F.; Tian, H.; Song, X. A selective and sensitive phthalimide-based fluorescent probe for hydrogen sulfide with a large stokes shift. RSC Advances 2015, 5, 98154-98159. [CrossRef]

45. Spectroscopic data for 13, derived from reaction of $\mathbf{6 a}$ and $7 \mathrm{a}:{ }^{1} \mathrm{H} \mathrm{NMR}\left(200 \mathrm{MHz}, \mathrm{CDCl}_{3}\right) \delta: 9.77(\mathrm{bs}, 1 \mathrm{H}$, $\mathrm{NH}), 8.69$ (d, J = 8.4 Hz, 1H, H-5), 7.64 (t, J = 7.9 Hz, 1H, H-6), 7.47 (d, J = 7.3 Hz, 1H, H-7), 4.29-4.11 (m, 1H, $\mathrm{CH}), 3.44\left(\mathrm{t}, J=6.6 \mathrm{~Hz}, 2 \mathrm{H}, \mathrm{CH}_{2} \mathrm{Cl}\right), 2.57\left(\mathrm{t}, J=7.1 \mathrm{~Hz}, 2 \mathrm{H}, \mathrm{CH}_{2} \mathrm{CO}\right), 2.34-2.24\left(\mathrm{~m}, 4 \mathrm{H}, \mathrm{CH}_{2}\right.$-cyclopentyl), 2.12-1.53 (m, 10H), 1.45-1.30 (m, 5H), $0.86\left(\mathrm{t}, J=7.4 \mathrm{~Hz}, 3 \mathrm{H}, \mathrm{CH}_{3}\right) .{ }^{13} \mathrm{C}$ NMR $\left(50 \mathrm{MHz}, \mathrm{CDCl}_{3}\right) \delta: 207.29$, $171.29,170.37,168.06,136.96,135.75,131.60,124.50,118.14,116.27,69.98,49.29,44.89,38.40,32.84,32.79,32.37$, 26.88, 26.33, 25.34 (2C), 23.30, 18.44, 11.42. ES-HRMS m/z for $\mathrm{C}_{24} \mathrm{H}_{31} \mathrm{ClN}_{2} \mathrm{O}_{4}[\mathrm{M}+\mathrm{H}]^{+}$: Calcd. 447.2051, found 447.2058 .

46. Ando, Y.; Niwa, K.; Yamada, N.; Irie, T.; Enomoto, T.; Kubota, H.; Ohmiya, Y.; Akiyama, H. Development of a Quantitative Bio/Chemiluminescence Spectrometer Determining Quantum Yields: Re-examination of the Aqueous Luminol Chemiluminescence Standard. Photochem. Photobiol. 2007, 83, 1205-1210. [CrossRef] [PubMed]

47. The reparation of a phosphonium-alkyl-acylaminophthalic acid turned out to be challenging, despite our repeated efforts. By employing 14 as reference, we attribute the diminished CL efficiency to the acyl moiety and not to the phosphonium cation. The existence of a great number of phosphonium-based fluorescent mito-trackers (ref. [29]) supports our assumption

48. Lee, J.; Seliger, H.H. Quantum yields of the luminol chemiluminescence reaction in aqueous and aprotic solvents. Photochem. Photobiol. 1972, 15, 227-237. [CrossRef]

49. Perrin, D.D.; Armarego, W.L.F. Purification of Laboratory Chemicals, 3rd ed.; Pergamon Press: Oxford, NY, USA, 1988.

50. Lohbeck, J.; Miller, A.K. Practical Synthesis of a Phthalimide-Based Cereblon Ligand to Enable Protac Development. Bioorg. Med. Chem. Lett. 2016, 26, 5260-5262. [CrossRef]

Sample Availability: Samples of the compounds are not available from the authors.

(C) 2019 by the authors. Licensee MDPI, Basel, Switzerland. This article is an open access article distributed under the terms and conditions of the Creative Commons Attribution (CC BY) license (http://creativecommons.org/licenses/by/4.0/). 